\title{
IS IT WISE TO KEEP LAMINATING?
}

\author{
MARC BRIANE $^{1}$ AND VINCENZO NESI ${ }^{2}$
}

\begin{abstract}
We study the corrector matrix $P^{\varepsilon}$ to the conductivity equations. We show that if $P^{\varepsilon}$ converges weakly to the identity, then for any laminate $\operatorname{det} P^{\varepsilon} \geq 0$ at almost every point. This simple property is shown to be false for generic microgeometries if the dimension is greater than two in the work Briane et al. [Arch. Ration. Mech. Anal., to appear]. In two dimensions it holds true for any microgeometry as a corollary of the work in Alessandrini and Nesi [Arch. Ration. Mech. Anal. 158 (2001) 155-171]. We use this property of laminates to prove that, in any dimension, the classical Hashin-Shtrikman bounds are not attained by laminates, in certain regimes, when the number of phases is greater than two. In addition we establish new bounds for the effective conductivity, which are asymptotically optimal for mixtures of three isotropic phases among a certain class of microgeometries, including orthogonal laminates, which we then call quasiorthogonal.
\end{abstract}

Mathematics Subject Classification. 35B27, 74Q15.

Received July 23, 2002.

\section{INTRODUCTION}

This paper is devoted to a detailed study of properties of sequences of solutions to conductivity equations in any dimension. The goal and the achievements will be described in the present section. In an attempt to a crude summary, the present paper studies the correctors to conductivity equations. In conjunction with the work [6], it shows, quite unexpectedly, that even in a simple linear conduction problem, in dimension greater than two, laminates and non laminates can be discriminated by a very simple property which will be stated later in the section. This property is always enjoyed by laminates but (as shown in [6]), not necessarily by non laminates.

The precise mathematical definitions will be reviewed briefly in Section 2. In the present section we will assume that the reader has already some familiarity with the notions of $G$ and $H$-convergence. We consider the following family of PDEs (conductivity problems) making the usual assumptions of the linear framework.

$$
\left\{\begin{aligned}
-\operatorname{div}\left(\sigma^{\varepsilon} \nabla u_{\varepsilon}\right)=f & & \text { in } \Omega \\
u_{\varepsilon}=g & & \text { on } \partial \Omega .
\end{aligned}\right.
$$

Keywords and phrases. Homogenization, bounds, composites, laminates.

1 Centre de Mathématique, INSA de Rennes \& IRMAR, 20 avenue des Buttes de Coësmes, 35043 Rennes Cedex, France; e-mail: mbriane@insa-rennes.fr

2 Dip. di Mat., Universitá di Roma, La Sapienza P.le A. Moro 2, 00185 Rome, Italy; e-mail: nesi@mat.uniroma1.it 
We consider the associated limiting problem

$$
\left\{\begin{aligned}
-\operatorname{div}\left(\sigma^{0} \nabla u_{0}\right)=f & & \text { in } \Omega \\
u_{0}=g & & \text { on } \partial \Omega .
\end{aligned}\right.
$$

The (symmetric) matrix $\sigma^{0}$ is called the $G$-limit (or the $H$-limit) of the sequence $\sigma^{\varepsilon}$. It is well known that, up to subsequences such a limiting problem exists and one has that

$$
\left\{\begin{aligned}
u_{\varepsilon} & \rightarrow u_{0} & & \text { weakly in } H^{1}(\Omega), \\
\sigma^{\varepsilon} \nabla u_{\varepsilon} & \rightarrow \sigma^{0} \nabla u_{0} & & \text { weakly in } L^{2}\left(\Omega ; \mathbb{R}^{d}\right) .
\end{aligned}\right.
$$

The convergence of the sequence $u_{\varepsilon}$ is not strong in general. For this reason one introduces the so called corrector matrix $P^{\varepsilon}$. which has the property that

$$
\nabla u_{\varepsilon}-P^{\varepsilon} \nabla u_{0} \longrightarrow 0 \quad \text { strongly in } L^{1}\left(\Omega ; \mathbb{R}^{d}\right)
$$

We will focus on proving properties of the corrector matrix for special sequences related to the so called laminates. We are interested in a class of $G$-closure problems which we now define. Given an integer $N \geq 3$ (called the number of phases), $N$ nonnegative numbers $p_{i}$ with $\sum_{i=1}^{N} p_{i}=1$ (called the volume fractions) and $N$ real numbers $0<\sigma_{1}<\cdots<\sigma_{N}$ (called the conductivities of the phases), consider the family of conductivity matrices

$$
\sigma^{\varepsilon}:=\sum_{i=1}^{N} \sigma_{i} \chi_{\varepsilon}^{i} I_{d} \quad \text { with } \quad \chi_{\varepsilon}^{i} \stackrel{L}{\infty}-w *_{\rightarrow}, \quad i=1, \ldots, N,
$$

where $L^{p}-w(*)$ denotes the $L^{p}$-weak $(*)$ convergence.

The goal is to describe the family of all possible limits $\sigma^{0}$ of the above sequences in some systematic way. Thanks to the localization principle we have (roughly speaking) that the $G$-closure can be represented by a finite dimensional space. We will make use of this principle only in this introduction.

Indeed set $p=\left(p_{1}, \ldots, p_{N}\right), \quad \sigma=\left(\sigma_{1}, \ldots, \sigma_{N}\right)$ and define $G=G(p, \sigma)$ as the closure of the set of constant and symmetric matrices $\sigma^{*}$ defined according to the following rule

$$
\sigma^{*}=\int_{Y} \sum_{i=1}^{N} \sigma_{i} X_{i}(y)\left(D U_{\sharp}(y)+I_{d}\right) \mathrm{d} y
$$

where $D U=\left[\frac{\partial U_{j}}{\partial y_{i}}\right]_{1 \leq i, j \leq d}$, the $X_{i}$ 's are characteristic functions of disjoint measurable subsets summing up to one, $Y=(0,1)^{d}$ and $U_{\sharp}$ is the solution to the cell problem

$$
\left\{\begin{array}{l}
\operatorname{div}\left(\sigma\left(\nabla U_{\sharp}+I\right)^{T}\right)=0 \text { in } \mathbb{R}^{d} \\
U_{\sharp} \in H_{\sharp}^{1}(Y)^{d} .
\end{array}\right.
$$

Then one proves that

$$
\sigma^{0}(x) \in G(\theta(x), \sigma) \text { a.e. in } \Omega \text {. }
$$

Therefore, for issues like establishing bounds it is often sufficient to consider periodic composites. The kind of questions we address in the present paper differ from the customary one and the more general approach seems the most appropriate.

When $N=2$, the $G$-closure is known. It is unknown for $N \geq 3$. This paper makes some progress in the latter problem. To state our result it is convenient to set up some notation. We identify the $G$-closure with one of its finite dimensional representations and call it $G$. A set $\mathcal{B}$ is called a bound for $G$ if $\mathcal{B} \supseteq G$. A point 
$B \in \partial \mathcal{B}$ is called attainable if there exists $\sigma^{*} \in G$ with $\sigma^{*}=B$. It is called optimal if there exists a sequence of the form (1.5) which $H$-converges to a constant $\sigma^{0} \in G$ with $\sigma^{0} \equiv B$. Notationally we will not distinguish the two cases and we simply write $M \in G$ in both cases. The latter are the usual definitions. Now we want to consider a family of $G$-closure problems. To fix ideas set $N=3$ and suppose that $p, \sigma_{1}$ and $\sigma_{2}$ are given. Regard $G$ and any bound on it as a function of $\sigma_{3}$ (the most conducting phase).

Definition 1.1. Assume that for any $\sigma_{3} \in\left[\sigma_{2}, \infty\right)$ there exists $M=M\left(\sigma_{3}\right) \in G\left(\sigma_{3}\right)$ and there exists a bound $\mathcal{B}\left(\sigma_{3}\right) \supseteq G\left(\sigma_{3}\right)$ such that

i) the sets $\mathcal{B}\left(\sigma_{3}\right)$ converge in the sense of Kuratowski to a set $\mathcal{B}(\infty)$, namely any convergent sequence (as $\sigma_{3}$ tends to infinity) $P\left(\sigma_{3}\right) \in \mathcal{B}\left(\sigma_{3}\right)$ converges to a point of $\mathcal{B}(\infty)$ and any point of $\mathcal{B}(\infty)$ is the limit of such a sequence;

ii) the sequence of matrices $M\left(\sigma_{3}\right)$ converge to a matrix $M(\infty)$;

iii) $M(\infty) \in \partial \mathcal{B}(\infty)$.

Then we say that the set $\mathcal{B}(\infty)$ is asymptotically optimal (A.O.) as $\sigma_{3}$ tends to infinity. We also say that any point of type $M(\infty)$ is A.O. Throughout the paper the sentence "as $\sigma_{3}$ tends to infinity" will be systematically omitted.

The set of all possible matrices $M(\infty)$ defined above is denoted by $A G$. In words our definition requires that the bounds approach the $G$-closure as $\sigma_{3}$ diverges. A mathematically more satisfactory approach requires an appropriate definition of the $G$-closure for composite with infinitely conducting phases. We believe that such an approach would fully motivate our definition. We will not pursue this idea in the present paper. Let us however remark that this definition is implicit in the work of Cherkaev [7], Gibiansky and Sigmund [9] and many others. We are now ready to explain our results.

\subsection{Dimension $d=2$}

We begin with dimension two where our analysis is based on the work of the second author and of Cherkaev to better motivate our work in dimension $d \geq 3$.

In dimension $d=2$ we consider a class of microgeometries proposed by Cherkaev [7] and prove A.O. for $N=3$ for a large (one dimensional) part of $\mathcal{B}(\infty)$. In our language A.O. was already established by Cherkaev [7] under the two following assumptions. First, the composite is isotropic, second the following (Cherkaev-condition) holds

$$
p_{2}\left(1-p_{2}\right) \sigma_{1}-p_{1}\left(1+p_{2}\right) \sigma_{2} \geq 0 .
$$

We extend his analysis to anisotropic composites showing that the condition above is not necessary. The set $\mathcal{B}(\infty)$ is unbounded above and it is described by the condition that in eigenvalue space the $A G$ has to lie above an unbounded curve which is shown in Section 3, Figure 3.

Our analysis shows that Cherkaev's microgeometries give some portion of this curve which has positive (actually infinite) length. If in addition (1.8) holds, one obtains that the whole curve belongs to $A G$ and hence the latter is fully characterized. It is important to note that the set $\mathcal{B}(\infty)$ is found using the bounds proved in [20] by the second author (see Sect. 3 for a review) that, in this regime are strictly tighter than the previously known one. Remark that the bounds do not reduce themselves to those of Hashin and Shtrikman [10] when the composites are isotropic. They are indeed tighter in certain regimes.

Our work complements the one by Gibiansky and Sigmund [9]. In their work they also look at what we call $A G$ and prove that, when

$$
p_{1}\left(\sigma_{1}+\sigma_{2}\right)-2\left(\sqrt{p_{2}}-p_{2}\right) \sigma_{1} \geq 0
$$

(Gibiansky-Sigmund regime), the Hashin-Shtrikman bounds for isotropic composites are A.O.

It can also be checked that there is still an intermediate regime where neither Cherkaev's nor GibianskySigmund condition holds. In this case we have only the following limited information. First, the best available bounds are again those by the second author. (So the work of Gibiansky and Sigmund was remarkably efficient 
giving the best possible regime of attainability for the Hashin-Shtrikman bounds.) Second, A.O. of the bounds in $[20]$ is not known.

In order to explain the other results of the present paper let us remark that the improved bounds in dimension $d=2$ hinge on the following fact which has an interest on its own.

$$
\text { If } d=2 \quad \text { and } \quad U_{\sharp} \text { is a solution of }(1.7) \text {, then } \operatorname{det}\left(D U_{\sharp}+I\right)>0 \text { a.e. }
$$

This result has been proved in [1] (see [2] for application to composites of the latter result). Similarly if $Y$ is replaced by any simply connected domain which is convex and the solution is searched for in $H_{0}^{1}$ rather than in $H_{\sharp}^{1}$, the same conclusion hold [1]. A weaker but equally useful result was previously proved in [3].

A corollary of any of these results can be obtained thank to the work of Tartar and Murat [17] yielding that for any sequence $\sigma^{\varepsilon}$ satisfying (1.5) and for which the $H$-limit $\sigma^{0}$ is constant, the corrector matrix converges to a constant matrix $P^{0}$ and it satisfies the following property in dimension $d=2$.

$$
\text { If } P^{\varepsilon} \rightarrow P^{0} \text { in } L^{2} \text {, with } \operatorname{det}\left(P^{0}\right)>0, \text { then } \operatorname{det}\left(P^{\varepsilon}\right) \geq 0 \text { a.e. }
$$

Let us summarize the results of dimension two. From (1.11) one obtains A.O. bounds for three phases (and actually also for $N>3$ phases in certain regimes).

\subsection{Dimension $\boldsymbol{d} \geq \mathbf{3}$}

We turn now our attention to dimensions $d \geq 3$. A natural question to ask is whether the following happens. For any sequence $\sigma^{\varepsilon}$ satisfying (1.5) and for which the $H$-limit $\sigma^{0}$ is constant, the corrector matrix converges to a constant matrix $P^{0}$ and it satisfies

$$
\text { if } d \geq 2 \text { and } P^{\varepsilon} \rightarrow P^{0} \text { in } L^{2} \text {, with } \mathrm{i}_{2}\left(P^{0}\right)>0, \quad \text { then } \quad \mathrm{i}_{2}\left(P^{\varepsilon}\right) \geq 0 \text { a.e., }
$$

where $i_{2}$ denotes the second invariant defined by

$$
\mathrm{i}_{2}(F):=\frac{1}{2}(\operatorname{Tr}(F))^{2}-\frac{1}{2} \operatorname{Tr}\left(F^{2}\right) .
$$

If this were the case, then new bounds in the spirit of [20], would easily be proved. However, we prove (see Prop. 2.14) that (1.12) is false in general for composites with more than two phases. More precisely, we show in Section 6.2 based on Example 2.17, that (1.12) is violated in any dimensions $d \geq 3$ by a rank-3 laminate made of three isotropic phases.

We proceed explaining our strategy. In dimension $d \geq 3$, we address a sub-problem of the $G$-closure one namely find the "lamination" closure. For precise definitions we refer to Section 2. Our strategy is very different from the previously most explored one. We ask the following question. Is it possible to establish rigorous bounds which have the following properties?

i) They are established only for laminates microstructures;

ii) they improve upon the classical bounds in a (sub)regime of the parameters where the Tartar-Murat bounds are not optimal.

We show that the answer is positive. The basic idea is to use the following higher dimensional analog of (1.11) for laminates microstructures (in the sense defined in Sect. 2.2.). Indeed, we prove (see Th. 2.13.) that the corrector matrix $P^{\varepsilon}$ associated to a laminate satisfies (1.11) in any dimension $d \geq 2$.

Remark that the corrector $P^{\varepsilon}$ is not uniquely defined. Our results holds when one chooses it according to the rule proved by the first author [5] (see Sect. 2).

An explicit improved bound using (1.11) can be written down using a slightly modified version of Tartar's method. However it requires a very long and tedious calculation. Instead of presenting the proof of this new bound, we present the proof of the following fact. 
Theorem 1.2. Consider a sequence $\sigma^{\varepsilon}$ satisfying (1.5) and let $\sigma^{0}$ be its $H$-limit. Then

(i) if, in some set of positive measure, the following condition holds true

$$
\left(\sum_{i=1}^{N} \frac{\theta_{i}}{\sigma_{i}+(d-1) \sigma_{1}}\right)^{d}<\sum_{i=2}^{N} \frac{\theta_{i}}{\left(\sigma_{i}+(d-1) \sigma_{1}\right)^{d}} \quad \text { a.e. },
$$

then the (Hashin-Shtrikman type) lower bound

$$
\frac{d}{\operatorname{Tr}\left(\sigma^{0}\right)+d(d-1) \sigma_{1}} \leq \sum_{i=1}^{N} \frac{\theta_{i}}{\sigma_{i}+(d-1) \sigma_{1}} \quad \text { a.e. }
$$

is not attained by a laminate.

(ii) Similarly, if the following condition holds true

$$
\left(\sum_{i=1}^{N} \frac{\theta_{i}}{\sigma_{i}+(d-1) \sigma_{N}}\right)^{d}<\sum_{i=1}^{N-1} \frac{\theta_{i}}{\left(\sigma_{i}+(d-1) \sigma_{N}\right)^{d}} \quad \text { a.e. }
$$

then the (Hashin-Shtrikman type) upper bound

$$
\frac{d}{d+(d-1) \sigma_{N} \operatorname{Tr}\left(\left(\sigma^{0}\right)^{-1}\right)} \leq \sum_{i=1}^{N} \frac{\theta_{i}}{1+(d-1) \frac{\sigma_{N}}{\sigma_{i}}} \quad \text { a.e. }
$$

is not attained by a laminate.

Remark 1.3. In fact the same arguments used to prove Theorem 1.2 can be generalized to show that the Hashin-Shtrikman type lower bound (4.1) obtained in $[13,15,23]$ is not attained by a laminate under the same regime (1.14).

An immediate consequence is that if the Hashin-Shtrikman bounds are optimal and (1.14) holds, then they are attained by non laminate structures. Our result should probably be interpreted as a very strong support to the non optimality in the given regime even among all microstructures.

Let us briefly digress to comment on the fact that in dimension $d \geq 3$ Milton and the authors [6] proved that there exists a (non laminate) periodic two-phase geometry such that the solution to the problem analogue to (1.7) but in dimension $d$ greater or equal to three, satisfies the following properties. It is defined almost everywhere and

$$
\left|\left\{x \in(0,1)^{d}: \operatorname{det}\left(D U_{\sharp}+I_{d}\right)>0\right\}\right|>0, \quad\left|\left\{x \in(0,1)^{d}: \operatorname{det}\left(D U_{\sharp}+I_{d}\right)<0\right\}\right|>0,
$$

where $|\cdot|$ denotes the $d$-dimensional Lebesgue measure. Therefore in higher dimensions there are microgeometries for which $\operatorname{det}\left(P^{\varepsilon}\right)$ changes sign and some for which it does not. Laminates always satisfy the latter property. This is a surprisingly simple instance showing once again that laminates cannot catch all the possible behaviors of general microstructures.

In the same spirit as before, we prove bounds for a subclass of composites which includes one that has been very successful so far, namely the class of orthogonal laminates. (The latter simply means that the lamination directions are always taken in a fixed orthonormal basis.) This class is not sufficient to obtain the full lamination closure. However, if one restricts attention to isotropic composites, we do not know of any example where this class does not suffices. For instance this class is sufficient when treating the $d$-dimensional $G$-closure of a polycrystal made of a single crystal, provided one restricts attention to isotropic composites! 
The new class we propose, which we call quasiorthogonal composites is the following slight modification of the class satisfying (1.12). We ask that the following property for the corrector $P^{\varepsilon}$ holds.

$$
\text { If } P^{\varepsilon} \rightarrow P^{0} \text { in } L^{2} \text {, with } \mathrm{i}_{2}\left(P^{0}\right) \geq 0 \text { a.e. then } \chi_{\varepsilon}^{1} \mathrm{i}_{2}\left(P^{\varepsilon}\right) \geq 0 \text { a.e. }
$$

We prove two facts about this class.

First, we prove A.O. bounds in any dimension and for any number of isotropic phases in appropriate regimes. The bounds are obtained using the same argument as in [20] and the A.O. is obtained with a straightforward extension to higher dimensions of Cherkaev's microgeometries.

Second, we prove that this class of composites contains the class of orthogonal laminates. This is the reason to name this class quasiorthogonal composites. Remark that, in dimension two, all composites with a constant $H$-limit are quasiorthogonal, but, in dimension $d>2$, there are composites which are not quasiorthogonal.

The paper is organized as follows. In Section 2 we review the necessary results from homogenization theory. We then state our main results about laminates, namely the positivity of the determinant of the corrector matrix, the quasiorthogonality of orthogonal laminates and the violation of (1.12). We also present some relevant examples of rank-three laminates.

In Section 3, we focus on dimension $d=2$. We review the known results in terms of bounds and we state our new one. In Section 4, we present known and new results in higher dimension. Section 5 is devoted to the proof of the bounds for quasiorthogonal composites in any dimension. Finally, in Section 6 we prove the result stated in Section 2.

The moral of the story in dimension greater than two is the following. Since we proved A.O. of Cherkaev's microgeometries among all possible quasiorthogonal laminates, one has the following consequence in addressing the $G$-closure problem. If one looks for new microgeometries with more extremal properties, then one is forced to restrict the search to non-quasiorthogonal composites. On the other hand, our incompatibility result for the Hashin-Shtrikman bounds show that either the bound need to be improved in that regime, or, if those bounds are optimal in the appropriate regime, then the corresponding microgeometries are not of a laminate type.

\section{HomogeniZATION AND LAMinAtion}

\subsection{Homogenization and $H$-convergence}

Notation 2.1. Let $d$ be a positive integer.

We denote by $\mathcal{M}_{d}$ the set of the $d \times d$ real matrices, by $\mathcal{M}_{d}^{s}$ its subset made of the symmetric matrices and by $I_{d}$ the identity matrix. $\mathbb{R}^{d}$ is provided with the usual scalar product denoted by $\cdot$ and with the associated norm denoted by $|\cdot|$.

$\mathcal{M}_{d}$ is provided with the scalar product : defined by $A: B:=\operatorname{Tr}\left(A^{T} B\right)$, where $\operatorname{Tr}$ is the trace and $A^{T}$ the transposed of $A$.

$\mathcal{M}_{d}$ is also provided with the norm $|\cdot|$ defined by $|A|:=(A: A)^{\frac{1}{2}}$.

Let $\kappa \geq 1$. We denote by $\mathcal{M}_{d}(\kappa)$ the subset of $\mathcal{M}_{d}^{s}$ made of the invertible matrices $A$ such that

$$
\forall \xi \in \mathbb{R}^{d}, \quad \min \left(A \xi \cdot \xi, A^{-1} \xi \cdot \xi\right) \geq \kappa^{-1}|\xi|^{2} .
$$

Note that any matrix $A \in \mathcal{M}_{d}(\kappa)$ satisfies $|A| \leq \kappa$.

Let $\Omega$ be a bounded open subset of $\mathbb{R}^{d}$.

Let $\varepsilon$ be a positive number.

Consider the following family of conduction problems:

$$
\left\{\begin{aligned}
-\operatorname{div}\left(\sigma^{\varepsilon} \nabla u_{\varepsilon}\right)=f & \text { in } \Omega \\
u_{\varepsilon}=g & \text { on } \partial \Omega,
\end{aligned}\right.
$$


where $\sigma^{\varepsilon}$ is a sequence of measurable matrix-valued functions from $\Omega$ into $\mathcal{M}_{d}(\kappa), f$ belongs to $H^{-1}(\Omega)$ and $g$ to $H^{1 / 2}(\Omega)$. The asymptotic behavior of problem (2.1) has been studied at depth. Spagnolo introduced the notion of $G$-convergence [21,22], Murat and Tartar that of $H$-convergence [16-18]. We will use the language of $H$-convergence which is the most appropriate for the problem we want to address.

One crucial result of the theory is the following.

Theorem 2.2 (Murat-Tartar). There exists a subsequence of $\varepsilon$ (still denoted by $\varepsilon$ ) and a measurable matrixvalued function $\sigma^{0}$ from $\Omega$ into $\mathcal{M}_{d}(\kappa)$ such that for any $f$ in $H^{-1}(\Omega)$ and $g$ in $H^{1 / 2}(\Omega)$, the following convergencies hold true

$$
\left\{\begin{aligned}
u_{\varepsilon} & \rightarrow u_{0} & & \text { weakly in } H^{1}(\Omega), \\
\sigma^{\varepsilon} \nabla u_{\varepsilon} & \rightarrow \sigma^{0} \nabla u_{0} & & \text { weakly in } L^{2}\left(\Omega ; \mathbb{R}^{d}\right),
\end{aligned}\right.
$$

where $u_{0}$ is the solution of problem (2.1) with $\varepsilon=0$.

Definition 2.3. When the weak convergencies (2.2) hold true for the whole sequence $\sigma^{\varepsilon}$ and for any right-hand sides $f$ and $g, \sigma^{\varepsilon}$ is said to $H$-converge to $\sigma^{0}$ and $\sigma^{0}$ is called the $H$-limit of $\sigma^{\varepsilon}$. In this case we write $\sigma^{\varepsilon} \stackrel{H}{\rightarrow} \sigma^{0}$. The $H$-convergence does not in general implies the strong convergence of $\nabla u_{\varepsilon}$ to $\nabla u_{0}$ in $L^{2}\left(\Omega, \mathbb{R}^{d}\right)$. In order to measure the oscillations of $\nabla u_{\varepsilon}$ around its weak limit $\nabla u_{0}$ one has to introduce a corrector term which is defined as follows.

Definition 2.4. Assume that $\sigma^{\varepsilon} \stackrel{H}{-} \sigma^{0}$. Let $P^{\varepsilon}$ be a matrix-valued function in $L^{2}\left(\Omega ; \mathcal{M}_{d}\right)$. The sequence $P^{\varepsilon}$ is said to be a corrector associated to $\sigma^{\varepsilon}$ if for any $f$ in $H^{-1}(\Omega)$ and $g$ in $H^{1 / 2}(\Omega)$, the solution $u_{\varepsilon}$ of problem (2.1) satisfies

$$
\nabla u_{\varepsilon}-P^{\varepsilon} \nabla u_{0} \longrightarrow 0 \quad \text { strongly in } L^{1}\left(\Omega ; \mathbb{R}^{d}\right) .
$$

Murat and Tartar [17] proved the following existence result.

Theorem 2.5 (Murat-Tartar). Assume that $\sigma^{\varepsilon} \stackrel{H}{-} \sigma^{0}$. Then there exists a corrector $P^{\varepsilon}$ associated to $\sigma^{\varepsilon}$.

In fact the existence of a corrector is deduced from the following compensated compactness properties due to Murat and Tartar [17]:

Theorem 2.6 (Murat-Tartar). Assume that the sequence $\sigma^{\varepsilon} H$-converges to $\sigma^{0}$ and let $P^{\varepsilon}$ be a bounded sequence in $L^{2}\left(\Omega ; \mathcal{M}_{d}\right)$ such that for any $\xi \in \mathbb{R}^{d}$,

$$
\left\{\begin{array}{l}
P^{\varepsilon} \xi \rightarrow \xi \text { weakly in } L^{2}\left(\Omega ; \mathbb{R}^{d}\right), \\
\operatorname{curl}\left(P^{\varepsilon} \xi\right) \text { is compact in } H^{-1}\left(\Omega ; \mathbb{R}^{d}\right), \\
\operatorname{div}\left(\sigma^{\varepsilon} P^{\varepsilon} \xi\right) \text { is compact in } H^{-1}(\Omega) .
\end{array}\right.
$$

Then $P^{\varepsilon}$ is a corrector associated to $\sigma^{\varepsilon}$ and the $H$-limit $\sigma^{0}$ of $\sigma^{\varepsilon}$ is given by the weak limit

$$
\sigma^{\varepsilon} P^{\varepsilon} \rightarrow \sigma^{0} \quad \text { weakly in } L^{2}\left(\Omega ; \mathcal{M}_{d}\right) \text {. }
$$

Remark 2.7. The $H$-convergence of $\sigma^{\varepsilon}$ and the corrector $P^{\varepsilon}$ do not depend on any subdomain of $\Omega$ but only on the given sequence $\sigma^{\varepsilon}$. The $H$-convergence is thus local.

\subsection{Lamination}

\subsubsection{Definitions}

In this section we will consider multiphase microstructures in which the conductivity matrix $\sigma^{\varepsilon}$ is constant in each phase, i.e.

$$
\sigma^{\varepsilon}:=\sum_{i \in I} \chi_{\varepsilon}^{i} \sigma^{i} \quad \text { a.e. in } \Omega,
$$


where $\sigma^{i}$ are matrices in $\mathcal{M}_{d}$ and $\chi_{\varepsilon}^{i}$ are measurable characteristic functions of disjoint sets making a partition of $\Omega$, such that

$$
\sum_{i \in I} \chi_{\varepsilon}^{i}=1 \quad \text { a.e. in } \Omega
$$

The sequence of characteristic functions carries geometric information and is often called microstructure. We will consider conductivity matrices $\sigma^{\varepsilon}$ of type (2.6) for which there exists a corrector $P^{\varepsilon}$ which has the same multiphase structure, i.e.

$$
P^{\varepsilon}:=\sum_{i \in I} \chi_{\varepsilon}^{i} P^{i}, \quad \text { where } P^{i} \in \mathcal{M}_{d} \text { are constant matrices. }
$$

This assumption is justified. Indeed for a large class of microstructures such a qualified corrector exists as proved by the first author in [5].

We will restrict ourselves to the class of microstructures called laminates.

Definition 2.8. Let $\widehat{\sigma}:=\left\{\sigma^{i}\right\}_{i \in I}$ be a finite family of $\mathcal{M}_{d}(\kappa)$, let $\varepsilon$ be a positive number and let $\xi$ be a unit-norm vector in $\mathbb{R}^{d}$. The matrix-valued function $\sigma^{\varepsilon}$ is said to be a rank-1 laminate of phases $\left\{\sigma^{i}\right\}_{i \in I}$, at the scale $\varepsilon$, in the direction $\xi\left(\xi \in \mathbb{R}^{d},|\xi|=1\right)$ if

$$
\sigma^{\varepsilon}(x):=\sum_{i \in I} X_{i}\left(\frac{\xi \cdot x}{\varepsilon}\right) \sigma^{i} \text { a.e. } x \in \Omega,
$$

where $X_{i}$ are 1-periodic measurable characteristic functions defined on $\mathbb{R}$.

We define $L_{1}^{\varepsilon}(\widehat{\sigma}):=\sigma^{\varepsilon}$.

Definition 2.9. Let $n$ be a positive integer, let $\varepsilon$ be a positive number and let $\xi^{1}, \ldots, \xi^{n}$ be $n$ unit-norm vectors in $\mathbb{R}^{d}$. The matrix-valued function $\sigma^{\varepsilon}$ is said to be a rank- $n$ laminate if it is defined by the following inductive procedure.

- At level $k=n$ and for any $j$ in a finite subset $J_{n}$ of $\mathbb{N}, \sigma_{n-1, j}^{\varepsilon}$ is a rank-1 laminate of constant phases $\sigma^{n, i} \in \mathcal{M}_{d}(\kappa)$, at the scale $\varepsilon^{n}$ and in the direction $\xi^{n}$.

- At level $k<n$ and for any $j$ in a finite subset $J_{k}$ of $\mathbb{N}, \sigma_{k-1, j}^{\varepsilon}$ is a rank- 1 laminate of phases which are either constant matrices in $\mathcal{M}_{d}(\kappa)$ or composites of type $\sigma_{k, i}^{\varepsilon}$ (defined at level $k$ ), at the scale $\varepsilon^{k}$ and in the direction $\xi^{k}$.

- At level $k=1, \sigma^{\varepsilon}$ is a rank-1 laminate of type $\sigma_{0}^{\varepsilon}$.

Let $\widehat{\sigma}:=\left\{\sigma^{i}\right\}_{i \in I}$ be the finite family of all the constant matrices in $\mathcal{M}_{d}(\kappa)$ which define the laminate. We then denote $\sigma^{\varepsilon}=L_{n}^{\varepsilon}(\widehat{\sigma})$.

We again restrict ourselves to a subclass of rank- $n$ laminates which have the following extra property.

Definition 2.10. Let $n$ be a positive integer. The matrix-valued function $\sigma^{\varepsilon}:=L_{n}^{\varepsilon}(\widehat{\sigma})$ is said to be an admissible rank- $n$ laminate if there exists a subset $\widehat{P}:=\left\{P^{i}\right\}_{i \in I}$ of $\mathcal{M}_{d}$ such that the matrix-valued function $P^{\varepsilon}:=L_{n}^{\varepsilon}(\widehat{P})$ is a corrector associated to $\sigma^{\varepsilon}$ (according to Def. 2.4.), which satisfies the following conditions. For any (constant or composite) phases $P_{k, i}^{\varepsilon}$ and $P_{k, j}^{\varepsilon}$ of the rank-n laminate $P^{\varepsilon}$ at level $k$, we have

$$
\left\{\begin{array}{l}
\bar{P}=I_{d}, \\
\bar{P}^{k, i}-\bar{P}^{k, j}=\xi^{k} \otimes \eta^{k, i, j}, \quad \text { where } \eta^{k, i, j} \in \mathbb{R}^{d}, \\
\left(\bar{Q}^{k, i}-\bar{Q}^{k, j}\right)^{T} \xi^{k}=0,
\end{array}\right.
$$


where $\bar{P}$ is the weak limit of $P^{\varepsilon}, \bar{P}^{k, i}$ the weak limit of $P_{k, i}^{\varepsilon}$ and $\bar{Q}^{k, i}$ the weak limit of $\sigma_{k, i}^{\varepsilon} P_{k, i}^{\varepsilon}$. Moreover we have

$$
\bar{Q}^{k, i}=\sigma_{k, i}^{0} \bar{P}^{k, i}, \quad \text { where } \sigma_{k, i}^{0} \in \mathcal{M}_{d}(\kappa) .
$$

Remark 2.11. Conditions (2.4) can be written as a large linear system the solutions of which are the matrices $P^{i}$ of $\widehat{P}$. The first condition in (2.10) corresponds to the first one in Theorem 2.6. The second condition of (2.10) takes in account the jump of the curl between $P_{k, i}^{\varepsilon}$ and $P_{k, j}^{\varepsilon}$. The third condition of (2.10) corresponds to the jump of the divergence between $\sigma_{k, i}^{\varepsilon} P_{k, i}^{\varepsilon}$ and $\sigma_{k, j}^{\varepsilon} P_{k, j}^{\varepsilon}$. The matrix $\sigma_{k, i}^{0}$ in (2.11) is the $H$-limit (in the sense of Def. 2.3) of the composite $\sigma_{k, i}^{\varepsilon}$ at level $k$ thanks to Theorem 2.6. The conditions which define the admissible rank- $n$ laminates are quite natural since the separation of scales allow us to consider simply the averaged quantities between two neighboring composites at any level of lamination. So in [5] (see also Ex. 2.16. below) the first author proves that, if at each level $k$ one considers the rank-1 laminate of a constant matrix $\sigma^{k}$ and a composite $\sigma_{k}^{\varepsilon}$, the Conditions (2.3) are satisfied and define a rank- $n$ laminate corrector in a unique way. But more general rank- $n$ laminations are admissible (see Ex. 2.17. below).

Finally we define the orthogonal laminates as follows.

Definition 2.12. A rank-n laminate is said to be orthogonal if the directions of lamination $\xi^{1}, \ldots, \xi^{n}$ belong to an orthonormal basis $\left(e^{1}, \ldots, e^{d}\right)$ of $\mathbb{R}^{d}$.

The rank-3 laminate of Example 2.17 is an orthogonal laminate (see Fig. 2).

\subsubsection{Properties of the laminates}

The most remarkable new property of the laminates that we prove is the following.

Theorem 2.13. Let $\sigma^{\varepsilon}=L_{n}^{\varepsilon}(\widehat{\sigma})$ be an admissible rank-n laminate and let $P^{\varepsilon}=L_{n}^{\varepsilon}(\widehat{P})$ its associated corrector. Then $\operatorname{det}\left(P^{\varepsilon}\right)$ is positive a.e. in $\Omega$.

Remark that, with our definition $P^{0}$ is the identity matrix.

It is natural to ask ourselves if the result of Theorem 2.13 can be extended to another matrix invariant and in particular to the (quadratic) second invariant defined by (1.13). There is no such an extension as shown by the following result.

Proposition 2.14. For any dimension $d \geq 3$, there exists a 3 -phases admissible rank-3 laminate which violates property (1.12).

However the situation is more favorable in the case of the orthogonal lamination.

Theorem 2.15. Orthogonal laminates with isotropic phases are quasiorthogonal in the sense of Definition 1.18.

\subsubsection{Examples}

This subsection is devoted to give some explicit examples of laminates. The aim is two-fold. First, we want to familiarize the reader with the notations giving them a geometric interpretation. Second, our Example 2.17 allows us to prove Proposition 2.14 showing that (1.12) does not hold in general.

Example 2.16. We consider the following 2-dimensional ( $d=2$ or $d=3$ for a cylindrical configuration) rank-3 laminate (Fig. 1).

Let $\xi^{1}, \xi^{2}, \xi^{3}$ be 3 unit-norm vectors in $\mathbb{R}^{d}$ and let $\chi_{\varepsilon}^{k}, k=1,2,3$, be the characteristic function defined by

$$
\chi_{\varepsilon}^{k}(x):=X_{k}\left(\frac{\xi^{k} \cdot x}{\varepsilon^{k}}\right) \quad \text { a.e. } x \in \Omega,
$$

where $X_{k}$ is a 1-periodic characteristic function of averaged-value $p_{k}$. 


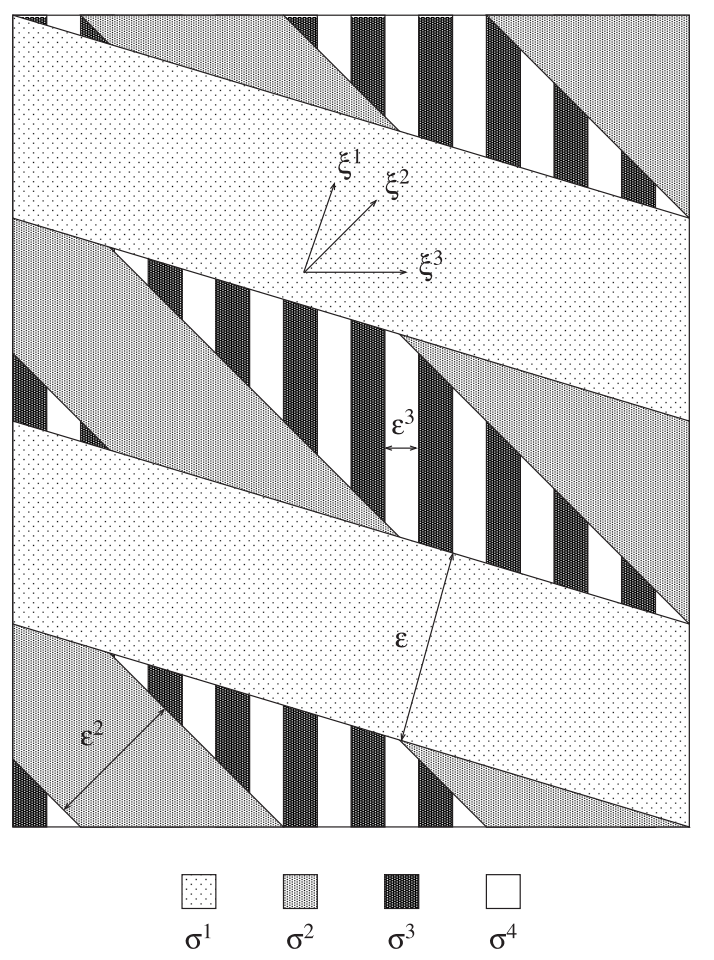

FiguRE 1. A $2 d$ rank-3 laminate.

Let $\widehat{\sigma}:=\left\{\sigma^{1}, \sigma^{2}, \sigma^{3}, \sigma^{4}\right\}$ be a family of 4 matrices in $\mathcal{M}_{d}(\kappa)$ and let us define the rank-3 laminate $\sigma^{\varepsilon}=$ $L_{3}^{\varepsilon}(\widehat{\sigma})$ by

$$
\sigma^{\varepsilon}:=\underbrace{\chi_{\varepsilon}^{1} \sigma^{1}+\left(1-\chi_{\varepsilon}^{1}\right)(\underbrace{\chi_{\varepsilon}^{2} \sigma^{2}+\left(1-\chi_{\varepsilon}^{2}\right)(\underbrace{\chi_{\varepsilon}^{3} \sigma^{3}+\left(1-\chi_{\varepsilon}^{3}\right) \sigma^{4}}_{\text {3rd rank-1 lamination }})}_{\text {2nd rank-1 lamination }})}_{\text {1st rank-1 lamination }} .
$$

The laminate $\sigma^{\varepsilon}$ is admissible and it is associated to the corrector $P^{\varepsilon}=L_{3}^{\varepsilon}(\widehat{\sigma})$ where $\widehat{P}:=\left\{P^{1}, P^{2}, P^{3}, P^{4}\right\}$ is defined by the following linear system deduced from Conditions (2.10):

$$
\left\{\begin{array}{l}
\bar{P}=p_{1} P^{1}+\left(1-p_{1}\right)\left(p_{2} P^{2}+\left(1-p_{2}\right)\left(p_{3} P^{3}+\left(1-p_{3}\right) P^{4}\right)\right)=I_{d} \\
P^{3}-P^{4}=\xi^{3} \otimes \eta^{3}, \\
P^{2}-\bar{P}^{2}=\xi^{2} \otimes \eta^{2}, \quad \bar{P}^{2}:=p_{3} P^{3}+\left(1-p_{3}\right) P^{4} \\
P^{1}-\bar{P}^{1}=\xi^{1} \otimes \eta^{1}, \quad \bar{P}^{1}:=p_{2} P^{2}+\left(1-p_{2}\right) \bar{P}^{2} \\
\left(\sigma^{3} P^{3}-\sigma^{4} P^{4}\right)^{T} \xi^{3}=0, \\
\left(\sigma^{2} P^{2}-\bar{Q}^{2}\right)^{T} \xi^{2}=0, \quad \bar{Q}^{2}:=p_{3} \sigma^{3} P^{3}+\left(1-p_{3}\right) \sigma^{4} P^{4} \\
\left(\sigma^{1} P^{1}-\bar{Q}^{1}\right)^{T} \xi^{1}=0, \quad \bar{Q}^{1}:=p_{2} \sigma^{2} P^{2}+\left(1-p_{2}\right) \bar{Q}^{2}
\end{array}\right.
$$


This system has a unique solution $\widehat{P}$ given by (see [5] for more details)

$$
\left\{\begin{array}{l}
P^{4}=\left(M^{1} M^{2} M^{3}\right)^{-1} \\
P^{3}=p_{3}^{-1}\left(\left(M^{1} M^{2}\right)^{-1}-\left(1-p_{3}\right)\left(M^{1} M^{2} M^{3}\right)^{-1}\right) \\
P^{2}=p_{2}^{-1}\left(\left(M^{1}\right)^{-1}-\left(1-p_{2}\right)\left(M^{1} M^{2}\right)^{-1}\right) \\
P^{1}=p_{1}^{-1}\left(I_{d}-\left(1-p_{1}\right)\left(M^{1}\right)^{-1}\right)
\end{array}\right.
$$

where the matrices $M^{k}, k=1,2,3$, are defined by

$$
\begin{gathered}
\left\{\begin{array}{l}
M^{3}:=I_{d}+\frac{p_{3}}{\sigma^{3} \xi^{3} \cdot \xi^{3}}\left(\xi^{3} \otimes \xi^{3}\right)\left(\sigma^{4}-\sigma^{3}\right), \\
M^{2}:=I_{d}+\frac{p_{2}}{\sigma^{2} \xi^{2} \cdot \xi^{2}}\left(\xi^{2} \otimes \xi^{2}\right)\left(\sigma_{2}^{0}-\sigma^{2}\right), \\
M^{1}:=I_{d}+\frac{p_{1}}{\sigma^{1} \xi^{1} \cdot \xi^{1}}\left(\xi^{1} \otimes \xi^{1}\right)\left(\sigma_{1}^{0}-\sigma^{1}\right),
\end{array}\right. \\
\text { and }\left\{\begin{array}{l}
\sigma_{2}^{0}:=\sigma^{3}+\left(1-p_{3}\right)\left(\sigma^{4}-\sigma^{3}\right)\left(M^{3}\right)^{-1} \\
\sigma_{1}^{0}:=\sigma^{2}+\left(1-p_{2}\right)\left(\sigma_{2}^{0}-\sigma^{2}\right)\left(M^{2}\right)^{-1}
\end{array}\right.
\end{gathered}
$$

In (2.14) $\sigma_{2}^{0}$ is the $H$-limit of the third rank-1 lamination of $\left\{\sigma^{3}, \sigma^{4}\right\}$ and $\sigma_{1}^{0}$ is the $H$-limit of the second rank-1 lamination of $\left\{\sigma^{2}, \sigma_{2}^{0}\right\}$. The $H$-limit of the rank-3 laminate $\sigma^{\varepsilon}$ defined by (2.12) is then equal to

$$
\sigma^{0}=\sigma^{1}+\left(1-p_{1}\right)\left(\sigma_{1}^{0}-\sigma^{1}\right)\left(M^{1}\right)^{-1}
$$

Example 2.17. We consider the following 3-dimensional rank-3 laminate (Fig. 2).

Let $\left(\xi^{1}, \xi^{2}, \xi^{3}\right)$ be an orthonormal basis of $\mathbb{R}^{3}$ and let $\chi_{\varepsilon}^{j}, j=1,2, \chi_{\varepsilon}^{3, k}, k=1,2,3$, be the characteristic functions defined by

$$
\left\{\begin{array}{l}
\chi_{\varepsilon}^{j}(x):=X_{j}\left(\frac{\xi^{j} \cdot x}{\varepsilon^{j}}\right) \\
\chi_{\varepsilon}^{3, k}(x):=X_{3, k}\left(\frac{\xi^{3} \cdot x}{\varepsilon^{3}}\right)
\end{array} \quad \text { a.e. } x \in \Omega,\right.
$$

where $X_{j}, X_{3, k}$, are 1-periodic characteristic functions of averaged-values $p_{j}, p_{3, k}$, such that $X_{3,1}+X_{3,2}+X_{3,3}=1$ a.e.

Let $\widehat{\sigma}:=\left\{\sigma^{1}, \sigma^{2}, \sigma^{3,1}, \sigma^{3,2}, \sigma^{3,3}\right\}$ be a family of five matrices in $\mathcal{M}_{3}(\kappa)$ and let us define the rank-3 laminate $\sigma^{\varepsilon}=L_{3}^{\varepsilon}(\widehat{\sigma})$ by

$$
\sigma^{\varepsilon}:=\underbrace{\chi_{\varepsilon}^{1} \sigma^{1}+\left(1-\chi_{\varepsilon}^{1}\right)(\underbrace{\chi_{\varepsilon}^{2} \sigma^{2}+\left(1-\chi_{\varepsilon}^{2}\right)(\underbrace{\chi_{\varepsilon}^{3,1} \sigma^{3,1}+\chi_{\varepsilon}^{3,2} \sigma^{3,2}+\chi_{\varepsilon}^{3,3} \sigma^{3,3}}_{\text {3rd rank-1 lamination }})}_{\text {2nd rank-1 lamination }})}_{\text {1st rank-1 lamination }} .
$$

In contrast to Example 2.17 the third rank-1 lamination is composed by three phases. This laminate is also admissible and the associated corrector $P^{\varepsilon}=L_{3}^{\varepsilon}(\widehat{P})$ with $\widehat{P}:=\left\{P^{1}, P^{2}, P^{3,1}, P^{3,2}, P^{3,3}\right\}$ is deduced from the 


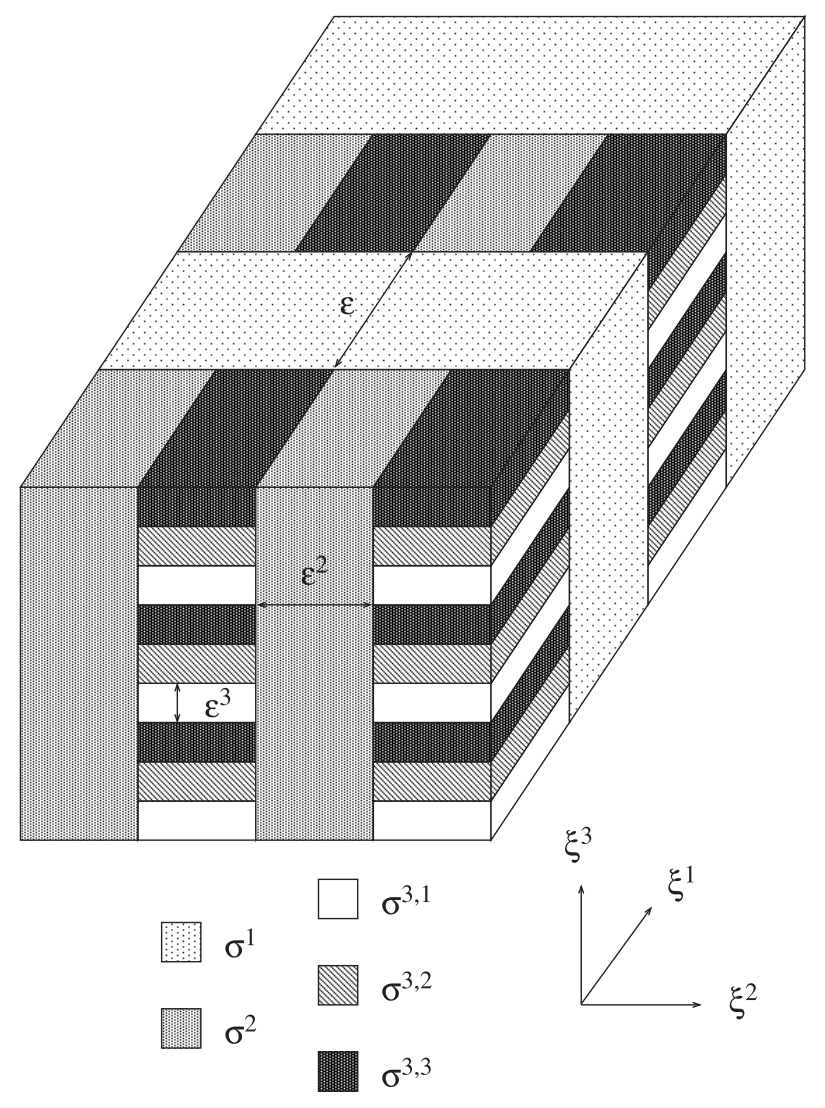

Figure 2. A 3-d rank-3 laminate.

following formulas similar to that of $(2.13)$ :

$$
\left\{\begin{array}{l}
P^{3, k}=\left(M^{1} M^{2} M^{3, k}\right)^{-1}, \quad k=1,2,3 \\
P^{2}=p_{2}^{-1}\left(\left(M^{1}\right)^{-1}-\left(1-p_{2}\right)\left(M^{1} M^{2}\right)^{-1}\right) \\
P^{1}=p_{1}^{-1}\left(I_{3}-\left(1-p_{1}\right)\left(M^{1}\right)^{-1}\right)
\end{array}\right.
$$

where the matrices $M^{1}, M^{2}, M^{3, k}, k=1,2,3$, are defined by

$$
\begin{aligned}
& \left\{\begin{array}{l}
M^{3, k}:=I_{3}+\sum_{j \neq k} \frac{p_{3, j}}{\sigma^{3, j} \xi^{3} \cdot \xi^{3}}\left(\xi^{3} \otimes \xi^{3}\right)\left(\sigma^{3, k}-\sigma^{3, j}\right), \quad k=1,2,3 \\
M^{2}:=I_{3}+\frac{p_{2}}{\sigma^{2} \xi^{2} \cdot \xi^{2}}\left(\xi^{2} \otimes \xi^{2}\right)\left(\sigma_{2}^{0}-\sigma^{2}\right), \\
M^{1}:=I_{3}+\frac{p_{1}}{\sigma^{1} \xi^{1} \cdot \xi^{1}}\left(\xi^{1} \otimes \xi^{1}\right)\left(\sigma_{1}^{0}-\sigma^{1}\right),
\end{array}\right. \\
& \text { and }\left\{\begin{array}{l}
\sigma_{2}^{0}:=\sigma^{3,1}+\sum_{j=2}^{3} p_{3, j}\left(\sigma^{3, j}-\sigma^{3,1}\right)\left(M^{3, j}\right)^{-1}, \\
\sigma_{1}^{0}:=\sigma^{2}+\left(1-p_{2}\right)\left(\sigma_{2}^{0}-\sigma^{2}\right)\left(M^{2}\right)^{-1}
\end{array}\right.
\end{aligned}
$$


In $(2.18) \sigma_{2}^{0}$ is the $H$-limit of the third rank-1 lamination of $\left\{\sigma^{3,1}, \sigma^{3,2}, \sigma^{3,3}\right\}$. Similarly to Example $2.16 \sigma_{1}^{0}$ is the $H$-limit of the second rank-1 lamination of $\left\{\sigma^{2}, \sigma_{2}^{0}\right\}$ and the $H$-limit of the rank-3 laminate $\sigma^{\varepsilon}(2.16)$ is also obtained by formula (2.15).

\section{WARMING-UP: STATEMENTS IN DIMENSION $d=2$}

Throughout the present section we consider composites made of a finite but arbitrary number of isotropic phases in prescribed volume fractions. In other words we assume that $\sigma_{\varepsilon}$ satisfies (1.5) and we denote by $\sigma^{0}$ its $H$-limit.

Theorem 3.1 (bounds: [20]). Assume

$$
\left(1-\theta_{2}\right) \sigma_{1}-\theta_{1} \sigma_{2}>0 \quad \text { a.e. }
$$

Then, the $H$-limit, $\sigma^{0}$ of a composite made of $N \geq 3$ isotropic phases with scalar conductivities $0<\sigma_{1}<\sigma_{2}<$ $\cdots<\sigma_{N}$ in prescribed volume fractions $\theta_{1}, \theta_{2}, \cdots, \theta_{N}$ satisfy the following bounds. For any $\lambda \in\left[\sigma_{1}, \sigma_{2}\right]$,

$$
\frac{\operatorname{Tr} \sigma^{0}-2 \lambda}{\operatorname{det} \sigma^{0}-\lambda^{2}} \leq 2 \sum_{i=1}^{N} \frac{\theta_{i}}{\sigma_{i}+\sigma_{i} \wedge \lambda} \quad \text { a.e. }
$$

The symbol $a \wedge b$ denotes the maximum between $a$ and $b$. Remark that for $\lambda=\sigma_{1}$ one obtains the LurieCherkaev [11], Murat-Tartar [23] bounds. If in addition the composite is isotropic the bound reduce to the original Hashin-Shtrikman one [10].

Proof. See [20].

To state the next results, recall the notion of A.O. in Definition 1.1.

Theorem 3.2 (A.O. for Hashin-Shtrikman: [9]). Under the hypotheses of Theorem 3.1 the bounds (3.2) specialized to the case $N=3, \lambda=\sigma_{1}$ and $\sigma^{0}=\lambda_{0} I_{d}$ are A.O. provided

$$
\theta_{1}\left(\sigma_{2}+\sigma_{1}\right)-2\left(\sqrt{\theta_{2}}-\theta_{2}\right) \sigma_{1} \geq 0 \quad \text { a.e. }
$$

Proof. See [9].

Remark 3.3. The result of Gibiansky and Sigmund is sharp in the following sense. When (3.3) does not hold and $\sigma_{3}=+\infty$, the bounds (3.2) specialized to the case $\sigma^{0}=\lambda_{0} I_{d}$, are strictly tighter than the Hashin-Shtrikman one.

We also need the following result due Milton [14] (see also [12] and [15]).

Theorem 3.4 (A.O. for anisotropic composites: [14]). The hypotheses of Theorem 3.1 in conjunction with

$$
\sigma_{1}^{0} \geq\left(\frac{\theta_{1}}{\sigma_{1}}+\frac{\theta_{2}}{\sigma_{2}}\right)^{-1} \quad \text { a.e. }
$$

fully characterize the AG-closure provided

$$
\theta_{1}\left(\sigma_{2}+\sigma_{1}\right)-2\left(1-\theta_{2}\right) \sigma_{1} \geq 0 \quad \text { a.e. }
$$

Proof. See [15].

Our new result in this context is the following. 


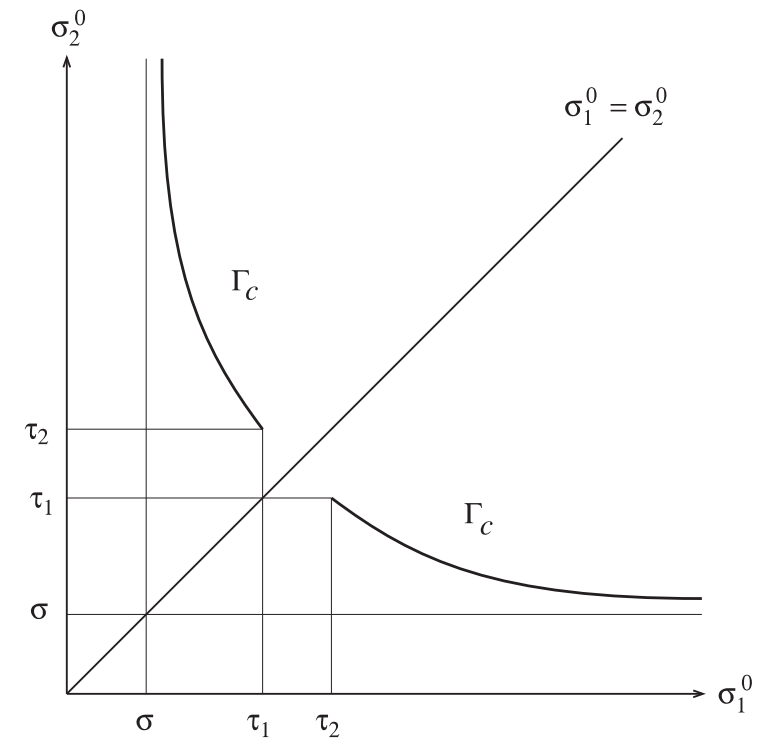

$$
\underline{\sigma}:=\left(\frac{\theta_{1}}{\sigma_{1}}+\frac{\theta_{2}}{\sigma_{2}}\right)^{-1}, \quad \tau_{1}:=\frac{\left(1-\theta_{2}\right)^{2}}{\theta_{1}} \sigma_{1}+\theta_{2} \sigma_{2}, \quad \tau_{2}:=\frac{\sigma_{2}}{\theta_{2}}
$$

Figure 3. The A.O. curve $\Gamma_{c}$.

Theorem 3.5 (attainment of (3.2 with $\left.\lambda=\sigma_{2}\right)$ ). Under the hypotheses of Theorem 3.1 the bounds (3.2) specialized to the case $N=3, \lambda=\sigma_{2}$ are A.O. More precisely, in eigenvalue space $\left(\sigma_{1}^{0}, \sigma_{2}^{0}\right)$, consider the (unbounded) curve $\Gamma$ defined by equality in (3.2) and $\lambda=\sigma_{2}$. Then its subset $\Gamma_{c}$ defined as

$$
\Gamma_{c}:=\Gamma \cap\left\{\left(\sigma_{1}^{0}, \sigma_{2}^{0}\right): \frac{\sigma_{2}}{\theta_{2}} \leq \max \left(\sigma_{1}^{0}, \sigma_{2}^{0}\right)\right\}
$$

is A.O.

For Figure 3, we have chosen $\theta_{1}, \theta_{2}, \sigma_{1}, \sigma_{2}$ such that Condition (3.7) does not hold or equivalently $\tau_{1}<\tau_{2}$. Any point of the curve $\Gamma_{c}$ in the eigenvalue space $\left(\sigma_{1}^{0}, \sigma_{2}^{0}\right)$, satisfies the equality in (3.2) and is asymptotically attained by a rank- 2 orthogonal laminate. The curve $\Gamma_{c}$ has a "hole" near the isotropic point $\left(2 \underline{\sigma}-\sigma_{2}, 2 \underline{\sigma}-\sigma_{2}\right)$. The "hole" is the region for which attainability (3.2) is not known.

Corollary 3.6 (Cherkaev [7]). If one has

$$
\theta_{2}\left(1-\theta_{2}\right) \sigma_{1}-\theta_{1}\left(1+\theta_{2}\right) \sigma_{2} \geq 0 \quad \text { a.e. }
$$

then $\Gamma_{c} \equiv \Gamma$ and the $A G$-closure is therefore characterized.

Remark 3.7. The exact statement proved by Cherkaev is that when (3.7) holds, the point on $\Gamma_{c}$ which satisfies $\sigma_{1}^{0}=\sigma_{2}^{0}$ is A.O. 


\section{The Core: Statements in Dimension $d \geq 3$}

As usual we assume that $\sigma_{\varepsilon}$ satisfies (1.5) and we denote by $\sigma^{0}$ its $H$-limit.

Theorem 4.1 (bounds: [13,15,23]). The H-limit $\sigma^{0}$ of a composite made of $N \geq 3$ isotropic phases with scalar conductivities $0<\sigma_{1}<\sigma_{2}<\cdots<\sigma_{N}$ in prescribed volume fractions $\theta_{1}, \theta_{2}, \ldots, \theta_{N}$ satisfy the following bounds:

$$
\frac{1}{\sigma_{1}+\left(\operatorname{Tr}\left(\sigma^{0}-\sigma_{1} I_{d}\right)^{-1}\right)^{-1}} \leq d \sum_{i=1}^{N} \frac{\theta_{i}}{(d-1) \sigma_{1}+\sigma_{i}}
$$

Proof. See [15].

Theorem 4.2 (A.O. for Hashin-Shtrikman: [14]). Under the hypotheses of Theorem 4.1, the bounds (4.1) specialized to the case $N=3$, in conjunction with (3.4), fully characterize the AG-closure provided

$$
\theta_{1}\left[\sigma_{2}+(d-1) \sigma_{1}\right]-d\left(1-\theta_{2}\right) \sigma_{1} \geq 0 \quad \text { a.e. }
$$

Proof. See [14]. A different derivation was found later in [12]. See also [15] for a review.

Remark 4.3. The two-dimensional result of Gibiansky and Sigmund [9] suggests that (4.2) is not sharp. Let us also emphasize that Milton, as well as Lurie and Cherkaev proved more. We have stated their result restricting attention to A.O.

Our new results in this context are the following. First, the negative result Theorem 1.2 stated in the introduction which will be proved in Section 5.3. On the positive side, we have a result of A.O. for quasiorthogonal composites which is the content of the next subsection.

\subsection{Bounds for quasiorthogonal composites}

Let us recall from the introduction that when there exists a corrector $P^{\varepsilon}$ which satisfies (1.18) we say that the microgeometry is quasiorthogonal. The definition is suggested by the fact that, as proved in Section 6.3, orthogonal laminates always satisfy it. Also, Proposition 2.14 shows that the class of quasiorthogonal microgeometries is strictly larger than that of orthogonal laminates. Let us also emphasize that, although we do know of laminates which are not quasiorthogonal, we are unable to prove that in this class one can find composites which lie outside the set prescribed by our bounds. In two dimensions, as already pointed out any microgeometry leading to a constant $H$-limit is quasiorthogonal.

Theorem 4.4 (bounds for quasiorthogonal composites). The $H$-limit $\sigma^{0}$ of a composite made of $N \geq 3$ isotropic phases in prescribed volume fractions and which, in addition, is quasiorthogonal satisfies the following bounds. Assume (3.1). Then, for any $t \in\left[\sigma_{1}, \sigma_{2}\right]$,

$$
\frac{1}{t+\left(\operatorname{Tr}\left(\sigma^{0}-t I_{d}\right)^{-1}\right)^{-1}} \leq d \sum_{i=1}^{N} \frac{\theta_{i}}{\sigma_{i}+(d-1) \sigma_{i} \wedge t} \quad \text { a.e. }
$$

The proof is presented in Section 5.1.

Theorem 4.5. Under the hypotheses of Theorem 4.4 the bounds (4.3) specialized to the case $N=3, \lambda=\sigma_{2}$ are A.O. More precisely, in eigenvalue space $\left(\sigma_{1}^{0}, \sigma_{2}^{0}, \ldots, \sigma_{d}^{0}\right)$, consider the (unbounded) surface $S$ defined by equality in (4.3) and $\lambda=\sigma_{2}$. Then, there exists a subset $S_{c}$ of $S$ of $(d-1)$-dimensional measure which is A.O. 


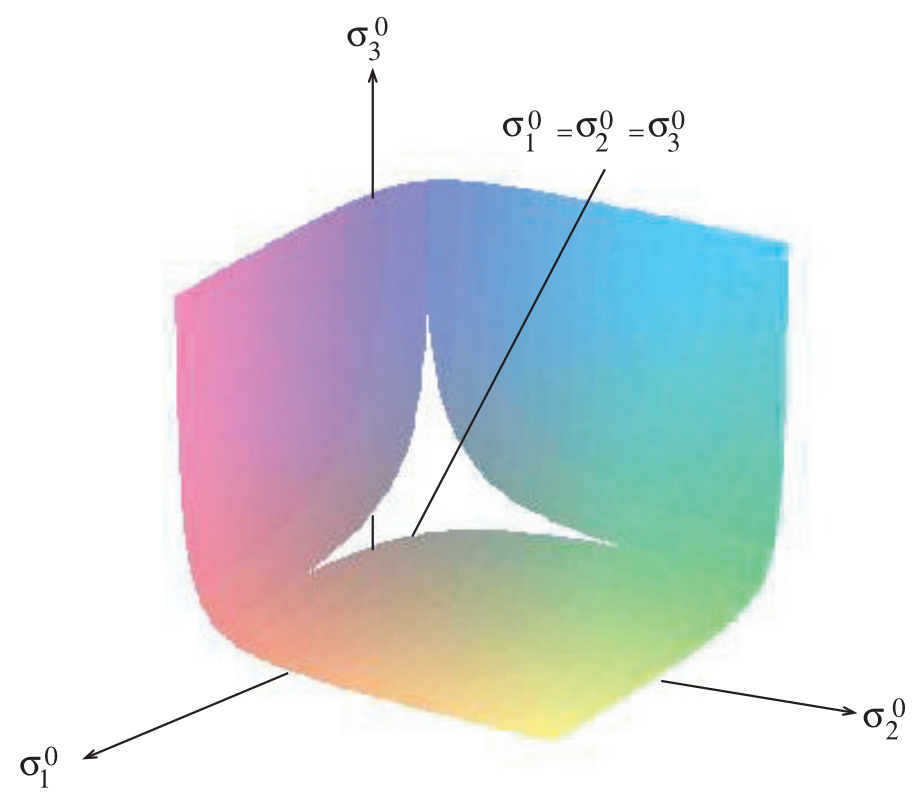

Figure 4. The A.O. surface $S_{c}$ with a bounded "hole" for $d=3$.

For Figure 4, we have chosen $d=3, \theta_{1}:=0.07, \theta_{2}:=0.4, \sigma_{1}:=1, \sigma_{2}:=2$ and $\sigma_{3}:=+\infty$ such that Condition (4.4) does not hold. Any point of the surface $S_{c}$ in the eigenvalue space $\left(\sigma_{1}^{0}, \sigma_{2}^{0}, \sigma_{3}^{0}\right)$, satisfies the equality in (4.3) and is asymptotically attained by a rank-3 orthogonal laminate. The surface $S_{c}$ does not "hit" the isotropic line $\sigma_{1}^{0}=\sigma_{2}^{0}=\sigma_{3}^{0}$. The unshaded triangle shaped "hole" is the region for which attainability (4.3) is not known.

Corollary 4.6 (Cherkaev's type result). If one has

$$
\theta_{2}\left(1-\theta_{2}\right) \sigma_{1}-\theta_{1}\left(d-1+\theta_{2}\right) \sigma_{2} \geq 0 \quad \text { a.e. }
$$

then $S_{c} \equiv S$ and the $A G$-closure is fully characterized.

\section{Proofs CONCERning BOUndS}

\subsection{Proof of Theorem 4.4}

We need some preliminary definitions and a few lemmas. Let $f: \mathcal{M} \times \mathcal{M} \times \mathcal{M}^{s} \times \mathbb{R} \rightarrow \mathbb{R}$ be defined by

$$
\begin{aligned}
f(S, A, B, t) & :=2 A: B-\operatorname{Tr}\left(A^{T} S A\right)-2 t \mathrm{i}_{2}(A), \\
g(S, B, t) & :=\sup _{A \in \mathcal{M}} f(S, A, B, t) .
\end{aligned}
$$

Lemma 5.1. Assume that $S=s I_{d}$ and $0<t<s$, then

$$
g\left(S, I_{d}, t\right)=\frac{d}{s+(d-1) t} .
$$

The proof is an elementary linear algebra exercise and it is omitted. 
Lemma 5.2 (Tartar [24]). Consider a sequence of conductivity matrices satisfying (1.5). Assume that $\sigma^{\varepsilon}$ is $H$-converging to $\sigma^{0}$. Let $P^{\varepsilon}$ be the corrector matrix associated to the conductivity $\sigma^{\varepsilon}$ and let $P^{0}$ be its $L^{2}$-weak limit. Then one has

$$
\forall \varphi \in C_{0}^{\infty}(\Omega), \varphi \geq 0, \quad \liminf _{\varepsilon \rightarrow 0^{+}} \int_{\Omega} \varphi f\left(\sigma^{\varepsilon}, P^{\varepsilon}, I_{d}, t\right) \mathrm{d} x \geq \int_{\Omega} \varphi f\left(\sigma^{0}, P^{0}, I_{d}, t\right) \mathrm{d} x .
$$

See [24].

Corollary 5.3. Under the same hypotheses of Lemma 5.2, set

$$
t_{\varepsilon}(x):=t_{1} \chi_{1}^{\varepsilon}(x)+t_{2} \sum_{i=2}^{N} \chi_{i}^{\varepsilon}(x) \quad \text { with } 0<t_{1}<\sigma_{1} \leq t_{2}<\sigma_{2} .
$$

If (1.18) holds, then for any $P^{0}$ with

$$
\mathrm{i}_{2}\left(P^{0}\right) \geq 0 \quad \text { a.e. }
$$

which in addition satisfies (1.18), one has

$$
\liminf _{\varepsilon \rightarrow 0^{+}} \int_{\Omega} \varphi g\left(\sigma^{\varepsilon}, I_{d}, t_{\varepsilon}\right) \geq \int_{\Omega} \varphi f\left(\sigma^{0}, P^{0}, I_{d}, t_{2}\right)
$$

Sketch of the proof. It is just a straightforward adaptation of Tartar's proof to the situation under consideration. At almost every point and for every admissible test function $\varphi$, one has

$$
\varphi g\left(\sigma^{\varepsilon}, I_{d}, t_{\varepsilon}\right) \geq \varphi f\left(\sigma^{\varepsilon}, P^{\varepsilon}, I_{d}, t_{\varepsilon}\right),
$$

hence

$$
\int_{\Omega} \varphi g\left(\sigma^{\varepsilon}, I_{d}, t_{\varepsilon}\right) \geq \int_{\Omega} \varphi f\left(\sigma^{\varepsilon}, P^{\varepsilon}, I_{d}, t_{\varepsilon}\right)=\int_{\Omega} \varphi f\left(\sigma^{\varepsilon}, P^{\varepsilon}, I_{d}, t_{2}\right)+2\left(t_{2}-t_{1}\right) \int_{\Omega} \varphi \chi_{\varepsilon}^{1} \mathrm{i}_{2}\left(P^{\varepsilon}\right) .
$$

Using (5.5), (5.6), and the assumption (1.18), one has

$$
2\left(t_{2}-t_{1}\right) \int_{\Omega} \varphi \chi_{\varepsilon}^{1} \mathrm{i}_{2}\left(P^{\varepsilon}\right) \geq 0 .
$$

Therefore, using Lemma 5.2,

$$
\liminf _{\varepsilon \rightarrow 0^{+}} \int_{\Omega} \varphi g\left(\sigma^{\varepsilon}, I_{d}, t_{\varepsilon}\right) \geq \liminf _{\varepsilon \rightarrow 0^{+}} \int_{\Omega} \varphi f\left(\sigma^{\varepsilon}, P^{\varepsilon}, I_{d}, t_{2}\right) \geq \int_{\Omega} \varphi f\left(\sigma^{0}, P^{0}, I_{d}, t_{2}\right) .
$$

Proof of Theorem 4.4. We compute both sides of inequality (5.7). Using Lemma 5.1 and the Definition 5.5 one has

Next choose

$$
\liminf _{\varepsilon \rightarrow 0^{+}} \int_{\Omega} \varphi g\left(\sigma^{\varepsilon}, I_{d}, t_{\varepsilon}\right)=d \int_{\Omega} \varphi\left[\frac{\theta_{1}}{\sigma_{1}+(d-1) t_{1}}+\sum_{i=2}^{N} \frac{\theta_{i}}{\sigma_{i}+(d-1) t_{2}}\right] .
$$

$$
P^{0}:=p_{0} I_{d}, \quad p_{0}:=\frac{d}{\operatorname{Tr} \sigma^{0}+d(d-1) t_{2}} .
$$

With the above choice, $P^{0}$ verifies (5.6) and therefore we can use (1.18). One has

$$
f\left(\sigma^{0}, p_{0} I_{d}, I_{d}, t_{2}\right)=\frac{d^{2}}{\operatorname{Tr} \sigma^{0}+d(d-1) t_{2}} .
$$


It follows that for all the admissible test functions $\varphi$,

$$
\int_{\Omega} \varphi\left[\frac{\theta_{1}}{\sigma_{1}+(d-1) t_{1}}+\sum_{i=2}^{N} \frac{\theta_{i}}{\sigma_{i}+(d-1) t_{2}}\right] \geq \int_{\Omega} \varphi\left[\frac{d^{2}}{\operatorname{Tr} \sigma^{0}+d(d-1) t_{2}}\right] .
$$

Finally sending $t_{1}$ to $\sigma_{1}$ and setting $t_{2}=t$ one obtains that for any $t \in\left[\sigma_{1}, \sigma_{2}\right]$, the following inequality holds

$$
\int_{\Omega} \varphi\left[\frac{\theta_{1}}{d \sigma_{1}}+\sum_{i=2}^{N} \frac{\theta_{i}}{\sigma_{i}+(d-1) t}\right] \geq \int_{\Omega} \varphi\left[\frac{d^{2}}{\operatorname{Tr} \sigma^{0}+d(d-1) t}\right] .
$$

The latter implies (4.3) when $\sigma^{0}=h_{0} I_{d}$ for some scalar $h_{0}$.

The more general case of anisotropic composites is handled similarly. We omit further algebraic details which can be found in [19].

Let us remark that our choice of the function $t_{\varepsilon}$ may appear rather mysterious. In fact better choices are possible for multiphase composites made by more than three phases. See [20] for more details. The main point however is that the new bounds, when evaluated in subregions of $\Omega$ where $\theta_{1}=0$, delivers an optimal bound (in contrast with the classical one) because it reduces itself to the two-phase bound.

\subsection{Proof of Theorem 4.2}

We explain the strategy due to Cherkaev in dimension $d$. Consider a rank- $d$ laminate. At the first step laminate all the $d$ phases along the coordinate direction $e^{d}$ in volume fractions $q_{1}, q_{2}, \ldots, q_{d}$ to be specified later. At the following $(d-1)$ steps, laminate in directions $e^{d-1}, \ldots, e^{1}$ respectively the previous composite with phase of conductivity $\sigma_{2} I_{d}$ with fractions $\beta_{d-1}, \ldots, \beta_{1}$. In this way one obtains a $(d-1)$-dimensional family of composites parameterized by the $\beta_{i}$ 's. One has to check that they are optimal. We give the details of the proof in two dimensions following Cherkaev. Extension to any dimension is straightforward.

We begin with a rank one laminate of the three given phases $\sigma_{1}, \sigma_{2}$ and $\sigma_{3}$ in volume fractions $q_{1}, q_{2}$ and $q_{3}$ respectively. Choose $e^{2}$ as direction of lamination. The effective conductivity of this "sub-composite" is diagonal and the explicit formulas for its eigenvalues are given by

$$
\lambda_{1}=q_{1} \sigma_{1}+q_{2} \sigma_{2}+q_{3} \sigma_{3}, \quad \lambda_{2}=\left(\frac{q_{1}}{\sigma_{1}}+\frac{q_{2}}{\sigma_{2}}+\frac{q_{3}}{\sigma_{3}}\right)^{-1} .
$$

Now laminate the latter subcomposite with $\sigma_{2} I_{2}$ in direction $e^{1}$ in volume fraction $1-\beta, \beta$ respectively. The resulting composite is a rank-two laminate. The final volume fractions of the three given phases are given by

$$
p_{1}=q_{1} \beta, \quad p_{3}=q_{3} \beta, \quad p_{2}=1-p_{1}-p_{3} .
$$

We choose $q_{i}>0$ with $\sum_{i=1}^{3} q_{i}=1$, so that

$$
(1-\beta)+\beta q_{2}=p_{2}
$$

We then regard each of the $q_{i}$ 's as a function of $\beta$. Their ranges imposes the constraint

$$
\beta \in\left[p_{1}+p_{3}, 1\right]
$$

as one easily verifies. The effective conductivity of the final (rank-two) composite is also diagonal and its eigenvalues are

$$
\nu_{1}=\left(\frac{\beta}{\lambda_{1}}+\frac{1-\beta}{\sigma_{2}}\right)^{-1}, \quad \nu_{2}=\beta \lambda_{2}+(1-\beta) \sigma_{2} .
$$


We regard the latter two as functions of $\beta$. As $\beta$ varies in its admissible range as given by (5.13), the pair $\left(\nu_{1}, \nu_{2}\right)$ describes a curve in eigenvalue space denoted by $\Gamma_{c}$ (see Fig. 3 ).

An easy calculation shows that

$$
\forall \beta \in\left(p_{1}+p_{3}, 1\right), \quad \lim _{\sigma_{3} \rightarrow+\infty}\left[\frac{\nu_{1}(\beta)+\nu_{2}(\beta)-2 \sigma_{2}}{\nu_{1}(\beta) \nu_{2}(\beta)-\sigma_{2}^{2}}-2\left(\frac{p_{1}}{2 \sigma_{1}}+\frac{p_{2}}{2 \sigma_{2}}+\frac{p_{3}}{\sigma_{2}+\sigma_{3}}\right)\right]=0 .
$$

Now we ask under which condition the curve $\Gamma_{c}$ intersects the diagonal or, in other words, under which condition the curve "hits" an isotropic composite. A lengthy but straightforward calculation that we omit shows that the exact condition is that

where $a$ and $b$ are defined by

$$
\beta_{C}:=\frac{2}{1-\sigma_{2}\left(\frac{1}{a}+\frac{1}{b \sigma_{2}^{2}}\right)}
$$

$$
a:=p_{1}\left(\sigma_{1}-\sigma_{2}\right)+p_{3}\left(\sigma_{3}-\sigma_{2}\right), \quad b:=\frac{p_{1}\left(\sigma_{2}-\sigma_{1}\right)}{\sigma_{1} \sigma_{2}}+\frac{p_{3}\left(\sigma_{2}-\sigma_{3}\right)}{\sigma_{3} \sigma_{2}} .
$$

When $\beta_{C} \in\left(p_{1}+p_{3}, 1\right)$, we set $\beta=\beta_{C}$. This implies, by construction, $\nu_{1}\left(\beta_{C}\right)=\nu_{2}\left(\beta_{C}\right)$ so that the rank-two laminate described before is indeed isotropic.

Next it is easy to check that in the limit as $\sigma_{3}$ tends to infinity, the condition on $\beta_{C}$ reduces to (3.7), i.e. to (4.4) specialized to the case $d=2$. Explicit calculation shows that $\Gamma_{c}$ reduces to the one stated in the theorem. In higher dimensions the A.O. surface can have a "hole" near the isotropic point in general (see Fig. 4). Notice that this hole can be also non-bounded and then the A.O. surface is not connected.

\subsection{Proof of Theorem $\mathbf{1 . 2}$}

The positivity of the determinant for the laminate correctors implies the incompatibility result stated in the introduction (Th. 1.2).

Proof of part (i). By localization we can assume that the homogenized matrix and the volumes fractions are constant. Let us consider the conductivity matrix $\sigma^{\varepsilon}$ defined by (1.5).

Let $F$ be the mapping defined on $\mathcal{M} \times \mathcal{M} \times \mathbb{R}$ by

$$
F(P, \sigma, t):=2 \operatorname{Tr} P-\operatorname{Tr}\left(P^{T} \sigma P\right)-2 t \mathrm{i}_{2}(P)
$$

where $\mathrm{i}_{2}$ is defined by (1.13), and let $G$ be the mapping defined on $\mathcal{M} \times \mathbb{R}$ by

$$
G(\sigma, t):=\sup _{P \in \mathcal{M}} F(P, \sigma, t) .
$$

A computation due to Tartar (Prop. 1 of [24]) yields for any $\sigma \geq t I_{d}$,

$$
G(\sigma, t)=\frac{1}{t+\left(\operatorname{Tr}\left(\sigma-t I_{d}\right)^{-1}\right)^{-1}}
$$

By applying Tartar's method [24] (see Lem. 5.2.) we obtain

$$
G\left(\sigma^{0}, \sigma_{1}\right) \leq \lim _{\mathcal{D}^{\prime}} G\left(\sigma^{\varepsilon}, \sigma_{1}\right)=\lim _{L^{\infty}-w *} G\left(\sigma^{\varepsilon}, \sigma_{1}\right) .
$$

Moreover by a convexity argument we have for any positive definite matrix $C$,

$$
\left(\operatorname{Tr}\left(C^{-1}\right)\right)^{-1} \leq d^{2} \operatorname{Tr} C .
$$


Inequalities (5.20) combined with (5.21) yield

$$
\frac{d^{2}}{\operatorname{Tr} \sigma^{0}+\left(d^{2}-d\right) \sigma_{1}} \leq G\left(\sigma^{0}, \sigma_{1}\right) \leq \lim _{L^{\infty}-w *} G\left(\sigma^{\varepsilon}, \sigma_{1}\right)=d \sum_{i=1}^{N} \frac{p_{i}}{\sigma_{i}+(d-1) \sigma_{1}}
$$

which implies the Hashin-Shtrikman bound (1.15).

Now let us assume that bound (1.15) is optimal. Then the inequalities (5.22) are all equalities. The left-hand side (in)equality of (5.22) implies that (5.21) is an equality for $C:=\sigma^{0}$, whence $\sigma^{0}=h I_{d}$. Let $P^{0}$ be a matrix such that $G\left(\sigma^{0}, \sigma_{1}\right)=F\left(P^{0}, \sigma^{0}, \sigma_{1}\right)$.

Let us also assume that $\sigma^{0}$ is attained by lamination, i.e. $\mathrm{x} \sigma^{\varepsilon}$ is a laminate, and let us consider the associated corrector $P^{\varepsilon}$ which weakly* converges to $P^{0}$ in $L^{\infty}$.

First, by compensated compactness and the definition of the homogenized matrix we have

$$
G\left(\sigma^{0}, \sigma_{1}\right)=F\left(P^{0}, \sigma^{0}, \sigma_{1}\right)=\lim _{\mathcal{D}^{\prime}} F\left(P^{\varepsilon}, \sigma^{\varepsilon}, \sigma_{1}\right)=\lim _{L_{\infty-w *}} F\left(P^{\varepsilon}, \sigma^{\varepsilon}, \sigma_{1}\right)
$$

since $P^{\varepsilon}$ and $\sigma^{\varepsilon}$ are bounded in $L^{\infty}$.

Second, we have

$$
F\left(P^{\varepsilon}, \sigma^{\varepsilon}, \sigma_{1}\right) \leq G\left(\sigma^{\varepsilon}, \sigma_{1}\right)=F\left(P_{\varepsilon}^{\prime}, \sigma^{\varepsilon}, \sigma_{1}\right)
$$

where $P_{\varepsilon}^{\prime}$ is a critical point of $F$, namely

$$
\left(\sigma^{\varepsilon}-\sigma_{1} I_{d}\right) P_{\varepsilon}^{\prime}+\sigma_{1} \operatorname{Tr}\left(P_{\varepsilon}^{\prime}\right) I_{d}=I_{d} \quad \text { a.e. in } \Omega \backslash\left\{\chi_{\varepsilon}^{1}=1\right\},
$$

which implies

$$
P_{\varepsilon}^{\prime}=P_{\varepsilon}^{\prime} \chi_{\varepsilon}^{1}+\sum_{i=2}^{N} \frac{1}{\sigma_{i}+(d-1) \sigma_{1}} I_{d} \chi_{\varepsilon}^{i} .
$$

Then the second (in)equality of (5.22) combined with (5.23) and (5.24) implies that $F\left(P_{\varepsilon}^{\prime}, \sigma^{\varepsilon}, \sigma_{1}\right)-F\left(P^{\varepsilon}, \sigma^{\varepsilon}, \sigma_{1}\right)$ is a non-negative sequence which weakly* converges to 0 in $L^{\infty}$ and thus strongly converges to 0 in $L^{2}$.

Now let $\omega_{\varepsilon}$ be a subdomain of the set where $\sigma^{\varepsilon}=\sigma_{i} I_{d}$, for $i>1$, of limit volume fraction $p>0$, and in which $P^{\varepsilon}$ takes a constant value $P$. We thus have the $L^{2}$-weak convergence

$$
1_{\omega_{\varepsilon}}\left(F\left(P^{\varepsilon}, \sigma^{\varepsilon}, \sigma_{1}\right)-F\left(P_{\varepsilon}^{\prime}, \sigma^{\varepsilon}, \sigma_{1}\right)\right) \rightarrow 0=p\left(F\left(P, \sigma_{i}, \sigma_{1}\right)-F\left(P_{i}^{\prime}, \sigma_{i}, \sigma_{1}\right)\right)
$$

which implies $F\left(P, \sigma_{i} I_{d}, \sigma_{1}\right)=F\left(P_{i}^{\prime}, \sigma_{i} I_{d}, \sigma_{1}\right)=G\left(\sigma_{i} I_{d}, \sigma_{1}\right)$. Then by the uniqueness of the maximum we obtain $P=P_{i}^{\prime}=\frac{1}{\sigma_{i}+(d-1) \sigma_{1}} I_{d}$. Therefore the laminate corrector also reads

$$
P^{\varepsilon}=P^{\varepsilon} \chi_{\varepsilon}^{1}+\sum_{i=2}^{N} \frac{1}{\sigma_{i}+(d-1) \sigma_{1}} I_{d} \chi_{\varepsilon}^{i}
$$

On the other hand, thanks to the positivity of the determinant of $P^{\varepsilon}$ we have

$$
\operatorname{det}\left(P^{\varepsilon}\right) \geq \sum_{i=2}^{N} \frac{1}{\left(\sigma_{i}+(d-1) \sigma_{1}\right)^{d}} \chi_{\varepsilon}^{i},
$$

whence by the rank- 1 affinity of the determinant

$$
\operatorname{det}\left(P^{0}\right)=\lim _{\mathcal{D}^{\prime}} \operatorname{det}\left(P^{\varepsilon}\right) \geq \sum_{i=2}^{N} \frac{p_{i}}{\left(\sigma_{i}+(d-1) \sigma_{1}\right)^{d}} .
$$


Moreover, since $G\left(\sigma^{0}, \sigma_{1}\right)=F\left(P^{0}, \sigma^{0}, \sigma_{1}\right), P^{0}$ is a critical point of $F\left(\cdot, \sigma^{0}, \sigma_{1}\right)$ whence $P^{0}=\frac{1}{h+(d-1) \sigma_{1}} I_{d}$. Finally bound (1.15) combined with inequality (5.28) yields

$$
\left(\sum_{i=1}^{N} \frac{p_{i}}{\sigma_{i}+(d-1) \sigma_{1}}\right)^{d} \geq \frac{1}{\left(h+(d-1) \sigma_{1}\right)^{d}} \geq \sum_{i=2}^{N} \frac{p_{i}}{\left(\sigma_{i}+(d-1) \sigma_{1}\right)^{d}}
$$

which contradicts Condition (1.14). Therefore the incompatibility of the optimality of the Hashin-Shtrikman lower bound (1.15) with the existence of an optimal laminate is proved.

Proof of part (ii). It is very similar to the part (i) by following Tartar's method [24] thanks to the mapping

$$
\tilde{F}(Q, \sigma, t):=(d-1) \operatorname{Tr}\left(Q^{T} Q\right)-(\operatorname{Tr} Q)^{2}-t(d-1) \operatorname{Tr}\left(Q^{T} \sigma^{-1} Q\right)+2 \operatorname{Tr} Q
$$

and the compact divergence sequence $Q_{\varepsilon}:=\sigma^{\varepsilon} P^{\varepsilon}$ which also has a non-negative determinant.

Remark 5.4. Condition (1.14) holds true for small enough volume fraction $p_{1}$ and for any choice of the values of the conductivities thanks to the strict convexity of the function $t \mapsto t^{d}$ in $[0,+\infty[$. Indeed set

$$
S\left(p_{1}\right):=\frac{1}{1-p_{1}} \sum_{i=2}^{N} \frac{p_{i}}{\sigma_{i}+(d-1) \sigma_{1}} \quad \text { and } \quad T\left(p_{1}\right):=\frac{1}{1-p_{1}} \sum_{i=2}^{N} \frac{p_{i}}{\left(\sigma_{i}+(d-1) \sigma_{1}\right)^{d}} .
$$

We have for fixed volume fractions $\frac{p_{i}}{1-p_{1}}, i>1$,

$$
\lim _{p_{1} \rightarrow 0}\left(p_{1} \frac{1}{d \sigma_{1}}+\left(1-p_{1}\right) S\left(p_{1}\right)\right)^{d}=S(0)^{d}<T(0)=\lim _{p_{1} \rightarrow 0}\left(1-p_{1}\right) T\left(p_{1}\right)
$$

whence (1.14) holds for small enough $p_{1}$.

\section{Proofs CONCERning PRoperties of LAminates}

\subsection{Proof of Theorem $\mathbf{2 . 1 3}$}

Let $P_{k, i}^{\varepsilon}$ be any (constant or composite) phase of $P^{\varepsilon}$ at the level $k$ of lamination, according to Definition 2.10, and let $\bar{P}^{k, i}$ be its $L^{\infty}$-weak* limit.

Let us first prove that for any $k, i, j$,

$$
\operatorname{det}\left(\bar{P}^{k, i}\right)=c_{k, i, j} \operatorname{det}\left(\bar{P}^{k, j}\right) \quad \text { where } c_{k, i, j}>0 .
$$

The Conditions (2.10) and (2.11) which are satisfied by $\bar{P}^{k, i}$ and $\bar{P}^{k, j}$ can be written in the following simpler way

$$
\bar{P}^{k, i}=M^{k, i, j} \bar{P}^{k, j} \quad \text { where } \quad M^{k, i, j}:=I_{d}+\frac{1}{\sigma_{k, i}^{0} \xi^{k} \cdot \xi^{k}}\left(\xi^{k} \otimes \xi^{k}\right)\left(\sigma_{k, j}^{0}-\sigma_{k, i}^{0}\right),
$$

and we easily check that

$$
\operatorname{det}\left(M^{k, i, j}\right)=\frac{\sigma_{k, j}^{0} \xi^{k} \cdot \xi^{k}}{\sigma_{k, i}^{0} \xi^{k} \cdot \xi^{k}}>0 .
$$

Therefore (6.1) holds true with $c_{k, i, j}:=\operatorname{det}\left(M^{k, i, j}\right)$.

Let us now prove by induction on the level $k$ of lamination, that for any $k, j, \operatorname{det}\left(\bar{P}^{k, j}\right)>0$. 
By Definition 2.10 at the first level of lamination $P^{\varepsilon}$ is a rank-1 laminate at scale $\varepsilon$ of the phases $\left\{P_{1, j}^{\varepsilon}\right\}_{j \in J_{1}}$ which are constant or composite phases at scales $\varepsilon^{2}, \ldots, \varepsilon^{n}$. Then thanks to the separation of scales in the weak convergence of oscillating sequences and by the first condition of (2.10) we have

$$
I_{d}=\sum_{j \in J_{1}} p_{1, j} \bar{P}^{1, j} \quad \text { where } \quad p_{1, j} \geq 0 \text { and } \sum_{j \in J_{1}} p_{1, j}=1
$$

and by the second condition of (2.10) we also have for any $i, j \in J_{1}, \bar{P}^{1, i}-\bar{P}^{1, j}=\xi^{1} \otimes \eta^{i, j}$. Therefore the rank-1 affinity of the determinant yields

$$
\operatorname{det}\left(I_{d}\right)=1=\sum_{j \in J_{1}} p_{1, j} \operatorname{det}\left(\bar{P}^{1, j}\right)
$$

which combined to (6.1) with $k=1$ implies that for any $j \in J_{1}$, $\operatorname{det}\left(\bar{P}^{1, j}\right)>0$. The result is thus satisfied for $k=1$.

Assume that the result holds true for $(k-1)$ and let $P_{k-1, i}^{\varepsilon}$ be a composite phase at level $k$. By Definition 2.10, $P_{k-1, i}^{\varepsilon}$ is a rank-1 laminate of the phases $\left\{P_{k, j}^{\varepsilon}\right\}_{j \in J_{k, i}}$. Then, similarly to the case $k=1$, by using the separation of scales, the rank-1 affinity of the determinant and the induction hypothesis, we obtain

$$
\operatorname{det}\left(\bar{P}^{k-1, i}\right)=\sum_{j \in J_{k, i}} p_{k, i, j} \operatorname{det}\left(\bar{P}^{k, j}\right)>0 \quad \text { where } \quad p_{k, i, j} \geq 0 \text { and } \sum_{j \in J_{k, i}} p_{k, i, j}=1
$$

which combined with (6.1), implies that for any $j \in J_{k, i}$, $\operatorname{det}\left(\bar{P}^{k, j}\right)>0$. The result is thus satisfied for $k$, which ends the induction proof.

Finally each constant phase $P^{i}$ in $\widehat{P}$ of the rank-n laminate $P^{\varepsilon}=L_{n}^{\varepsilon}(\widehat{P})$ is equal to some matrix $\bar{P}^{k, j}$ and thus has a positive determinant. Therefore since $P^{\varepsilon}$ can be written

$$
P^{\varepsilon}=\sum_{i \in I} \chi_{\varepsilon}^{i} P^{i}
$$

where $\chi_{\varepsilon}^{i}$ are characteristic functions whose the sum is equal to 1 , we obtain

$$
\operatorname{det}\left(P^{\varepsilon}\right)=\sum_{i \in I} \chi_{\varepsilon}^{i} \operatorname{det}\left(P^{i}\right)>0 \quad \text { a.e. in } \Omega
$$

which concludes the proof of Theorem 2.13 .

\subsection{Proof of Proposition $\mathbf{2 . 1 4}$}

Let us start by the dimension $d=3$. Let us consider the rank-3 laminate of Example 2.16 with isotropic phases $\sigma^{k}:=\sigma_{k} I_{3}, k=1, \ldots, 4$ and $\sigma_{4}=\sigma_{2}$. This laminate is a 3 -phases composite in dimension $d=3$. We define the second and third directions of lamination by $\xi^{2}:=\left(\frac{1}{\sqrt{2}}, \frac{1}{\sqrt{2}}, 0\right)$ and $\xi^{3}:=(1,0,0)$, the first one will be chosen later. 
By Definition (2.14) we have

$$
\left\{\begin{array}{l}
M_{3}=I_{3}+a\left(\xi^{3} \otimes \xi^{3}\right) \quad \text { where } a:=\frac{p_{3}}{\sigma_{3}}\left(\sigma_{2}-\sigma_{3}\right)>0 \\
M_{2}=I_{3}-\xi^{2} \otimes \xi^{2}+O\left(1-p_{2}\right)+O\left(\frac{\sigma_{3}}{\sigma_{2}}\right)+O\left(\left(1-p_{3}\right) \frac{\left(\sigma_{2}+\sigma_{3}\right)}{\sigma_{2}}\right) \\
M_{1}=I_{3}-\xi^{1} \otimes \xi^{1}+O\left(1-p_{1}\right)+O\left(\frac{\sigma_{2}}{\sigma_{1}}\right)+O\left(\left(1-p_{2}\right) \frac{\left(\sigma_{1}+\sigma_{2}+\sigma_{3}\right)}{\sigma_{1}}\right)
\end{array}\right.
$$

Set

$$
M:=\left(I_{3}-\xi^{2} \otimes \xi^{2}\right)\left(I_{3}+a\left(\xi^{3} \otimes \xi^{3}\right)\right) .
$$

We can then choose $\sigma_{3} \ll \sigma_{2} \ll \sigma_{1}$ and $1-p_{k} \ll 1$ for $k=1,2,3$, in such a way that

$$
\left\{\begin{array}{l}
M_{3}=I_{3}+a\left(\xi^{3} \otimes \xi^{3}\right) \quad \text { with } a \gg 1 \\
M_{2}=I_{3}-\xi^{2} \otimes \xi^{2}+o(1) \\
M_{1}=I_{3}-\xi^{1} \otimes \xi^{1}+o(1) \\
M_{2} M_{3}=M+o(1) \\
M_{1} M_{2} M_{3}=M-\left(\xi^{1} \otimes \xi^{1}\right) M+o(1)
\end{array}\right.
$$

where $o(1)$ means a small term in a given matrix norm.

Let $M^{S}$ be the symmetrized of the matrix $M$ defined by (6.4) and let $\lambda_{1} \leq \lambda_{2} \leq \lambda_{3}$ be the eigenvalues of $M^{S}$. We can check that

$$
\begin{gathered}
M=\frac{1}{2}\left(\begin{array}{ccc}
1+a & -1-a / 2 & 0 \\
-1-a / 2 & 1 & 0 \\
0 & 0 & 2
\end{array}\right) \\
\text { and } \lambda_{1}=\frac{1-\sqrt{2}}{4} a+o(a), \quad \lambda_{2}=1, \quad \lambda_{3}=\frac{1+\sqrt{2}}{4} a+o(a) .
\end{gathered}
$$

Now we choose $\xi^{1}$ as the unit-norm eigenvector associated to the eigenvalue $\lambda_{3}$. By the previous estimates we have

$$
\operatorname{Tr}\left(M_{1} M_{2} M_{3}\right)=\operatorname{Tr}\left(M^{S}\right)-M^{S} \xi^{1} \cdot \xi^{1}+o(1)=\lambda_{1}+\lambda_{2}+o(1),
$$

which implies that for $a$ large enough $\operatorname{Tr}\left(M_{1} M_{2} M_{3}\right)<0$.

Finally, by the Definition (2.13) of the last phase $P^{4}$ of the corrector $P^{\varepsilon}$ and the Definition (1.13) of the second invariant, we obtain

$$
\mathrm{i}_{2}\left(P^{4}\right)=\operatorname{det}\left(P^{4}\right) \operatorname{Tr}\left(M_{1} M_{2} M_{3}\right)<0 .
$$

Therefore the second invariant of the rank-3 laminate $P^{\varepsilon}$ is not non-negative a.e. in $\Omega$, which concludes the proof of Proposition 2.14.

Example 6.1. Numerically we computed the following values.

1) Let us consider the 3-rank and 3-phases laminate defined as follows. The directions of lamination are $\xi^{1}:=\left(t, \sqrt{1-t^{2}}, 0\right)$ with $t:=-0.9, \xi^{2}:=\left(\frac{1}{\sqrt{2}}, \frac{1}{\sqrt{2}}, 0\right), \xi^{3}:=(1,0,0)$, the layers volume fractions are $p_{1}:=0.95$, $p_{2}=p_{3}:=0.9$ and the layers conductivity are $\sigma^{1}:=10^{3} I_{3}, \sigma^{2}=\sigma^{3}:=10 I_{3}, \sigma^{3}:=10^{-1} I_{3}$.

We obtain that the corrector in the fourth layer satisfies

$$
P^{4}=\left(\begin{array}{ccc}
0.4280983664 & -0.1566620987 & 0 \\
17.98398313 & -3.673461792 & 0 \\
0 & 0 & 1
\end{array}\right) \quad \text { and } \quad \mathrm{i}_{2}\left(P^{4}\right)=-2.000557878<0
$$


2) Let us consider the 3-rank and 3-phases laminate defined as follows. The directions of lamination are $\xi^{1}:=$ $\left(t, \sqrt{1-t^{2}}, 0\right)$ with $t:=0.3, \xi^{2}:=\left(\frac{1}{\sqrt{2}}, \frac{1}{\sqrt{2}}, 0\right), \xi^{3}:=(1,0,0)$, the layers volume fractions are $p_{1}=p_{2}=p_{3}:=0.9$ and the layers conductivity are $\sigma_{1}:=10^{3} I_{3}, \sigma_{2}=\sigma_{4}=10^{-1} I_{3}, \sigma_{3}:=10^{2} I_{3}$.

We obtain that the corrector in the fourth layer satisfies

$$
P^{4}=\left(\begin{array}{ccc}
-8.331725368 & -51.35846901 & 0 \\
0.01643576281 & 0.07230966407 & 0 \\
0 & 0 & 1
\end{array}\right) \quad \text { and } \quad \mathrm{i}_{2}\left(P^{4}\right)=-8.017764352<0 .
$$

Remark 6.2. In the previous counter-examples the geometry has a cylindrical symmetry so that it is essentially 2-dimensional because the directions of lamination belong to the same plane.

Let us prove now that the result of Proposition 2.14 also holds for dimension $d>3$. We will do this thank to a slight modification of the 3-dimensional counter-example. Let $\sigma_{3}^{\varepsilon}$ be the 3 -dimensional laminate of the first example in Example 6.1 and $P_{3}^{\varepsilon}$ its associated corrector. Set

$$
\sigma^{\varepsilon}:=\left(\begin{array}{cc}
\sigma_{3}^{\varepsilon} & 0 \\
0 & I_{d-3}
\end{array}\right) \quad \text { and } \quad P^{\varepsilon}:=\left(\begin{array}{cc}
P_{3}^{\varepsilon} & 0 \\
0 & I_{d-3}
\end{array}\right) .
$$

It is easy to see that $\sigma^{\varepsilon}$ is a $d$-dimensional laminate and $P^{\varepsilon}$ its associated corrector. By Example 6.1 we know that the constant value $P_{3}^{4}$ of the fourth phase of $P_{3}^{\varepsilon}$ satisfies $\mathrm{i}_{2}\left(P_{3}^{4}\right)<0$ and we can also choose $\operatorname{Tr}\left(P_{3}^{4}\right)<0$ as large we want. Then the constant value $P^{4}$ of the fourth phase of $P^{\varepsilon}$ satisfies according to (6.5),

$$
\begin{aligned}
\mathrm{i}_{2}\left(P^{4}\right) & =\frac{1}{2}\left(\operatorname{Tr}\left(P_{3}^{4}\right)+d-3\right)^{2}-\frac{1}{2}\left(\operatorname{Tr}\left(P_{3}^{4}\right)^{2}+d-3\right) \\
& =\mathrm{i}_{2}\left(P_{3}^{4}\right)+(d-3)\left(\operatorname{Tr}\left(P_{3}^{4}\right)+\frac{d-4}{2}\right)<0,
\end{aligned}
$$

whence the non-positivity of the second invariant for any dimension $d \geq 3$.

\subsection{Proof of Theorem $\mathbf{2 . 1 5}$}

Let $\sigma^{\varepsilon}=L_{n}^{\varepsilon}(\widehat{\sigma})$ be an orthogonal admissible rank- $n$ laminate such that the family $\widehat{\sigma}$ is composed of isotropic phases, i.e. $\widehat{\sigma}=\left\{\sigma_{i} I_{d}\right\}_{i \in I}$. We can assume that the directions of lamination belong to the canonic basis of $\mathbb{R}^{d}$.

First, let us prove by a decreasing induction on $k$ that any matrix $\sigma_{k, i}^{0}$ defined by (2.11) is diagonal.

The result holds true for $k=n$ since by Definition 2.10 at level $n$ any matrix $\sigma_{n, i}^{0}$ is equal to a matrix of $\widehat{\sigma}$ and is thus isotropic.

Assume that the result holds true for $k$. Let $\sigma_{k-1, i}^{0}$ be a matrix at level $k$ of lamination. By Definition 2.10 and Remark $2.11 \sigma_{k-1, i}^{0}$ is the $H$-limit of the sequence $\sigma_{k-1, i}^{\varepsilon}$ which is a rank-1 lamination at scale $\varepsilon^{k}$ of the phases $\left\{\sigma_{k, j}^{\varepsilon}\right\}_{j \in J_{k, i}}$, i.e.

$$
\sigma_{k-1, i}^{\varepsilon}(x):=\sum_{j \in J_{k, i}} X_{k, j}\left(\frac{\xi^{k} \cdot x}{\varepsilon^{k}}\right) \sigma_{k, j}^{\varepsilon}, \quad \text { a.e. } x \in \Omega,
$$

where $X_{k, j}$ are 1-periodic characteristic functions. By the principle of reiterated homogenization (see e.g. [4]) the matrix $\sigma_{k-1, i}^{0}$ is also the $H$-limit of the same rank-1 lamination (6.6) with the phases $\left\{\sigma_{k, j}^{0}\right\}_{j \in J_{k, i}}$, where each matrix $\sigma_{k, j}^{0}$ is the $H$-limit of $\sigma_{k, j}^{\varepsilon}$ obtained by a homogenization process at scales $\varepsilon^{k+1}, \ldots, \varepsilon^{n}$. Then the homogenization formula for rank-1 lamination in direction $\xi^{k}$ yields

$$
\sigma_{k-1, i}^{0}=\sum_{j \in J_{k, i}} p_{k, j} \sigma_{k, j}^{0}\left(I_{d}+\sum_{h \neq j} \frac{p_{k, h}}{\sigma_{k, h}^{0} \xi^{k} \cdot \xi^{k}}\left(\xi^{k} \otimes \xi^{k}\right)\left(\sigma_{k, j}^{0}-\sigma_{k, h}^{0}\right)\right),
$$


where each $p_{k, j}$ is the averaged-value of the characteristic function $X_{k, j}$ in (6.6). By the induction hypothesis each matrix $\sigma_{k, j}^{0}$ is diagonal as well as the product $\left(\xi^{k} \otimes \xi^{k}\right)$ by the orthogonal lamination assumption. Therefore formula (6.7) implies that the matrix $\sigma_{k-1, i}^{0}$ is also diagonal and positive since $\sigma_{k-1, i}^{0} \in \mathcal{M}_{d}(\kappa)$. The result is thus satisfied for $(k-1)$, which ends the induction proof.

Second, let us prove by an increasing induction on $k$ that any matrix $\bar{P}^{k, i}$ defined in (2.10) is positive diagonal. Let us start by the following preliminary remark. Since any product $\left(\xi^{k} \otimes \xi^{k}\right)$ is positive diagonal as well as any matrix $\sigma_{k, i}^{0}$ by the previous result, it is easy to check that any matrix $M^{k, i, j}$ defined by (6.2) is also positive diagonal. Let us now come back to the induction proof.

For $k=1$ and by $(6.2),(6.3)$ we have for any $i, j$,

$$
\bar{P}^{1, i}=M^{1, i, j} \bar{P}^{1, j} \quad \text { and } \quad I_{d}=\sum_{j \in J_{1}} p_{1, j} \bar{P}^{1, j} \quad \text { where } \quad p_{1, j} \geq 0, \sum_{j \in J_{1}} p_{1, j}=1 .
$$

These equalities combined with $M^{1, i, j}$ positive diagonal clearly imply that any matrix $\bar{P}^{1, i}$ is positive diagonal. The result is thus satisfied for $k=1$.

Assume that it holds true for $(k-1)$. By the rule $(6.2)$ and by the definition of the rank-1 lamination at level $k$, any matrix $\bar{P}^{k-1, i}$ is associated to a family $\left\{\bar{P}^{k, j}\right\}_{j \in J_{k, i}}$ such that for any $j_{1}, j_{2} \in J_{k, i}$,

$$
\bar{P}^{k, j_{1}}=M^{k, j_{1}, j_{2}} \bar{P}^{1, j_{2}} \quad \text { and } \quad \bar{P}^{k-1, i}=\sum_{j \in J_{k, i}} p_{k, j} \bar{P}^{k, j} \quad \text { where } \quad p_{k, j} \geq 0, \sum_{j \in J_{k, i}} p_{k, j}=1 .
$$

Since the matrix $\bar{P}^{k-1, i}$ is positive diagonal by the induction hypothesis as well as any matrix $M^{k, j_{1}, j_{2}}$, we deduce from the previous equalities that any matrix $\bar{P}^{k, j}$ is positive diagonal. The result is thus satisfied for $k$, which ends the induction proof.

Finally, any matrix $P^{i}$ in $\widehat{P}$, which is of type $\bar{P}^{k, j}$, is positive diagonal. Therefore the corrector $P^{\varepsilon}=L_{n}^{\varepsilon}(\widehat{P})$ is positive diagonal a.e. in $\Omega$. In particular, all its invariants are positive a.e. in $\Omega$, which concludes the proof of Theorem 2.15 .

Acknowledgements. This research was partly carried out when V. Nesi was visiting the Centre de Mathématiques at the INSA de Rennes and the Centre de Mathématiques Appliquées at the École polytechnique.

\section{REFERENCES}

[1] G. Alessandrini and V. Nesi, Univalent $\sigma$-harmonic mappings. Arch. Ration. Mech. Anal. 158 (2001) 155-171.

[2] G. Alessandrini and V. Nesi, Univalent $\sigma$-harmonic mappings: applications to composites. ESAIM: COCV 7 (2002) 379-406.

[3] P. Bauman, A. Marini and V. Nesi, Univalent solutions of an elliptic system of partial differential equations arising in homogenization. Indiana Univ. Math. J. 50 (2001) (Spring).

[4] A. Bensoussan, J.L. Lions and G. Papanicolaou, Asymptotic Analysis for Periodic Structures. North-Holland (1978).

[5] M. Briane, Correctors for the homogenization of a laminate. Adv. Math. Sci. Appl. 4 (1994) 357-379.

[6] M. Briane, G.W. Milton and V. Nesi, Change of sign of the corrector's determinant in three dimensions. Arch. Ration. Mech. Anal. To appear.

[7] A. Cherkaev, Variational methods for structural optimization. Appl. Math. Sci. 140 (2000).

[8] A. Cherkaev and L.V. Gibiansky, Extremal structures of multiphase heat conducting composites. Internat J. Solids Structures 33 (1996) 2609-2618.

[9] L.V. Gibiansky and O. Sigmund, Multiphase composites with extremal bulk modulus. J. Mech. Phys. Solids 48 (2000) $461-498$.

[10] Z. Hashin and S. Shtrikman, A variational approach to the theory of effective magnetic permeability of multiphase materials. J. Appl. Phys. 33 (1962) 3125-3131.

[11] K.A. Lurie and A.V. Cherkaev, Exact estimates of conductivity of composites formed by two isotropically conducting media taken in prescribed proportions. Proc. R. Soc. Edinb. A 99 (1984) 71-87.

[12] K.A. Lurie and A.V. Cherkaev, The problem of formation of an optimal isotropic multicomponent composite. J. Opt. Theory Appl. 46 (1985) 571-589. 
[13] K.A. Lurie and A.V. Cherkaev, Exact estimates of the conductivity of a binary mixture of isotropic materials. Proc. R. Soc. Edinb. A 104 (1986) 21-38.

[14] G.W. Milton, Concerning bounds on transport and mechanical properties of multicomponent composite materials. Appl. Phys A 26 (1981) 125-130.

[15] G.W. Milton and R.V. Kohn, Variational bounds on the effective moduli of anisotropic composites. J. Mech. Phys. Solids 36 (1988) 597-629.

[16] F. Murat, Compacité par compensation : condition nécessaire et suffisante de continuité faible sous une hypothèse de rang constant. Ann. Scuola Norm. Sup. Pisa Cl. Sci. 4 (1981) 69-102.

[17] F. Murat, H-convergence. Séminaire d'Analyse Fonctionnelle et Numérique (1977-78), Université d'Alger. English translation: Murat F. and Tartar L., H-convergence. Topics in the Mathematical Modelling of Composite Materials, L. Cherkaev and R.V. Kohn Ed., Birkaüser, Boston, Progr. Nonlinear Differential Equations Appl. (1998) 21-43.

[18] F. Murat and L. Tartar, Calcul des variations et homogénéisation, in Les Méthodes de l'homogénéisation : théorie et applications en physique. Eyrolles (1985) 319-369.

[19] V. Nesi, Using quasiconvex functionals to bound the effective conductivity of composite materials. Proc. R. Soc. Edinb. Sect. A 123 (1993) 633-679.

[20] V. Nesi, Bounds on the effective conductivity of $2 d$ composites made of $n \geq 3$ isotropic phases in prescribed volume fractions: the weighted translation method. Proc. R. Soc. Edinb. A 125 (1995) 1219-1239.

[21] S. Spagnolo, Sul limite delle soluzioni di problemi di Cauchy relativi all'equazione del calore. Ann. Scuola Norm. Sup. Pisa 3 (1967) 657-699.

[22] S. Spagnolo, Sulla convergenza di soluzioni di equazioni paraboliche ed ellittiche. Ann. Scuola Norm. Sup. Pisa 3 (1968) 571-597.

[23] L. Tartar, Estimations de coefficients homogénéisés. Lect. Notes Math. 704 (1978) 364-373. English translation: Estimations of homogenized coefficients, in Topics in the mathematical modelling of composite materials. Progr. Nonlinear Differ. Equ. Appl. 31 (1997) 9-20.

[24] L. Tartar, Estimations fines des coefficients homogénéisés, in Ennio De Giorgi's Colloquium, Paris, 1983, P. Kree Ed., Pitman, Boston (1985) 168-187.

[25] L. Tartar, Compensated compactness and applications to p.d.e. in nonlinear analysis and mechanics, Heriot-Watt Symposium, Vol. IV, R.J. Knops Ed., Pitman, Boston (1979) 136-212. 